\title{
Extraordinary Doping Effects on Quasiparticle Scattering and Bandwidth in Iron-Based Superconductors
}

\author{
Z. R. Ye, ${ }^{1}$ Y. Zhang, ${ }^{1}$ F. Chen, ${ }^{1}$ M. Xu,${ }^{1}$ J. Jiang, ${ }^{1}$ X. H. Niu, ${ }^{1}$ C. H. P. Wen, ${ }^{1}$ L. Y. Xing, ${ }^{2}$ X. C. Wang, ${ }^{2}$ \\ C. Q. Jin, ${ }^{2}$ B. P. Xie, ${ }^{1, *}$ and D. L. Feng ${ }^{1, \dagger}$ \\ ${ }^{1}$ State Key Laboratory of Surface Physics, Department of Physics, and Advanced Materials Laboratory, \\ Fudan University, Shanghai 200433, People's Republic of China \\ ${ }^{2}$ Institute of Physics, Chinese Academy of Sciences, Beijing 100190, China \\ (Received 28 April 2014; revised manuscript received 13 July 2014; published 4 September 2014)
}

\begin{abstract}
The diversities in crystal structures and ways of doping result in extremely diversified phase diagrams for iron-based superconductors. With angle-resolved photoemission spectroscopy, we have systematically studied the effects of chemical substitution on the electronic structure of various series of iron-based superconductors. Beyond the Fermi-surface alteration that has been reported most often in the past, we found two more extraordinary effects of doping: (1) the site and band dependencies of quasiparticle scattering and, more importantly, (2) the ubiquitous and significant change of electronic correlation by both isovalent and heterovalent dopants in the iron-anion layer. Moreover, we found that the electronic correlation could be suppressed by applying either the chemical pressure or doping electrons but not by doping holes. Together with other findings provided here, these results complete the microscopic picture of the electronic effects of dopants, which facilitates a unified understanding of the diversified phase diagrams and resolutions to many open issues of various iron-based superconductors.
\end{abstract}

DOI: 10.1103/PhysRevX.4.031041

\section{INTRODUCTION}

Chemical substitution, or doping, is the most common way to tune the properties of a correlated material. The dopants can affect the electronic properties of materials in three ways: (1) by changing the chemical potential, which alters the Fermi surface, (2) by acting as scattering centers, which changes the electronic dynamics [1], and (3) by tuning the electronic hopping integral or screening strength, which would affect the overall electronic correlations [2,3].

The high-temperature superconductivity in cuprates is induced by doping a few percent of additional holes or electrons into their insulating antiferromagnetic parent compounds. Similarly, the domelike superconducting regions are reached in the phase diagrams of most ironbased high-temperature superconductors (FeHTS's) by doping their metallic collinear-antiferromagnetic (CAF) parent compounds as well $[4,5]$. However, unlike the cuprates where superconductivity only emerges with carrier doping, for FeHTS's, whether it is doped with heterovalent elements to introduce holes [e.g., $\mathrm{Ba}_{1-x} \mathrm{~K}_{x} \mathrm{Fe}_{2} \mathrm{As}_{2}$ (Ref. [6])]

\footnotetext{
*bpxie@fudan.edu.cn

†dlfeng@fudan.edu.cn
}

Published by the American Physical Society under the terms of the Creative Commons Attribution 3.0 License. Further distribution of this work must maintain attribution to the author(s) and the published article's title, journal citation, and DOI.
Subject Areas: Condensed Matter Physics,

Superconductivity or electrons [e.g., $\mathrm{Ba}\left(\mathrm{Fe}_{1-x} \mathrm{Co}_{x}\right)_{2} \mathrm{As}_{2}$ (Ref. [7])], or it is doped with isovalent elements to introduce compressional [e.g., $\mathrm{BaFe}_{2}\left(\mathrm{As}_{1-x} \mathrm{P}_{x}\right)_{2}$ (Refs. [8,9])] or tensile [e.g., $\mathrm{Ba}\left(\mathrm{Fe}_{1-x} \mathrm{Ru}_{x}\right)_{2} \mathrm{As}_{2}$ (Ref. [10])] strain, the generic features of the phase diagrams, such as a superconducting dome, are qualitatively the same. More intriguingly, differing from the universal doping range observed in cuprates [11], the sizes of the superconducting domes vary significantly in various families of FeHTS's [6-10,12-15]. For example, the superconductivity in $\mathrm{Ba}\left(\mathrm{Fe}_{1-x} \mathrm{Co}_{x}\right)_{2} \mathrm{As}_{2}$ disappears at $12 \%$ electron doping [7], while $\mathrm{LaFeAsO}_{1-x} \mathrm{H}_{x}$ shows superconductivity at the doping level as high as $40 \%$ [16].

In addition to the issues related to the overall phase diagram, there are various other unexplained doping behaviors as well. First, in $\mathrm{Ba}\left(\mathrm{Fe}_{1-x} \mathrm{Co}_{x}\right)_{2} \mathrm{As}_{2}$, through electron doping, the central pockets change from hole type to electron type, known as the Lifshitz transition, which was found to be accompanied by the disappearance of superconductivity [17]. The nesting between the hole and electron pockets was also suggested to be responsible for the maximal superconducting transition temperatures $\left(T_{C}\right.$ 's) in $\mathrm{Ba}\left(\mathrm{Fe}_{1-x} \mathrm{Co}_{x}\right)_{2} \mathrm{As}_{2}$ and $\mathrm{Ba}_{1-x} \mathrm{~K}_{x} \mathrm{Fe}_{2} \mathrm{As}_{2}$ (Ref. [18]). However, their counterexamples have been raised in iron-chalcognides [19-21], and the role of the Fermi-surface topology on superconductivity is still an open debate. Second, the superconductivity of FeHTS's seems to be much less sensitive to various common impurities in comparison to the cuprates [22,23]. Taking 
$\mathrm{Ba}\left(\mathrm{Fe}_{1-x} \mathrm{Co}_{x}\right)_{2} \mathrm{As}_{2}$ as an example, though the cobalt (Co) dopants are in the iron (Fe) layer, the maximal $T_{C}$ is as high as $22 \mathrm{~K}$ for $8 \%$ doping [24]. Such robustness of $T_{C}$ was proposed to be important for understanding the pairing symmetry of superconductivity $[25,26]$. However, the underlying microscopic mechanism of impurity scattering in FeHTS's is still unclear. Third, there is also an empirical relationship between $T_{C}$ and the anion-Fe-anion bond angle or height of the anion with respect to the Fe layer (referred to as anion height). It was found that $T_{C}$ is maximized when the bond angle is around $109.47^{\circ}$ or the anion height is around $1.38 \AA[27,28]$. However, so far, the direct connection among the lattice, electronic structure, and $T_{C}$ is yet to be established. All these unusual and seemingly unrelated puzzles require a deeper and more comprehensive understanding of the doping effects in FeHTS's. The diversity of the materials and diversified ways of doping add complexities to the task; however, they also provide an opportunity because a systematical study of various series of FeHTS's would help to pin down the common and critical ingredients of the unconventional superconductivity in these compounds.

Here, we present our systematic study of the doping effects on the electronic structures of the so-called 11, 111, and 122 series of FeHTS's with angle-resolved photoemission spectroscopy (ARPES). For the three essential consequences of doping - tuning the Fermi surface, scattering quasiparticles, and changing the electronic correlation in FeHTS's - our data reveal many extraordinary behaviors of the latter two consequences, helping us to answer many current unresolved issues and puzzles related to the doping, and unify the diversified phase diagrams. More specifically, we discovered the following: (1) The quasiparticle scattering induced by the dopants exhibits a band-selective and site-dependent behavior. All the bands, except the $d_{x y}$ holelike band around the zone center, are inert to the doped impurities. Moreover, the scattering strength of the $d_{x y}$ holelike band depends on the site of the dopants. The dopants at the Fe site cause the strongest scattering, and those at the anion site cause sizable scattering, while those off the Fe-anion plane do not cause much scattering. (2) The bandwidth observed by ARPES reflects the strength of the electronic correlation. We found that both the heterovalent doping and isovalent doping cause dramatic changes to the observed bandwidth. Remarkably, the Co dopants at the $\mathrm{Fe}$ site cause the strongest bandwidth enhancement, and phosphorus (P) or tellurium (Te) dopants at the anion site increase the bandwidth moderately, while the potassium $(\mathrm{K})$ dopants off the $\mathrm{Fe}$-anion plane do not noticeably affect the bandwidth. We further found that such doping evolutions of electronic correlation are due to the change of lattice structure, such as bond length and the type of filling carriers. Meanwhile, the latter affects the electronic correlation in a particle-hole asymmetric fashion, which highlights the distinctive Hund's rule coupling character of the electronic correlations in FeHTS's. (3) The Fermi-surface topology in FeHTS's shows a large diversity. We further demonstrate that the disappearance of certain hole pockets does not have to correspond to a diminishing $T_{C}$. Moreover, we found that, for the heavily electron-doped compounds with the same Fermi-surface topology, only systems with moderate electronic correlations exhibit superconductivity.

Many previous studies have tried to establish the relationship between $T_{C}$ and the Fermi-surface topology. However, many of these attempts, such as the Fermi-surface nesting scenario for optimizing $T_{C}$, have been proven to be just accidental in some peculiar compounds [19-21,29]. In the present paper, we further point out that the Fermi-surface topology is drastically different for various FeHTS's, and it likely plays a secondary role in the superconductivity of FeHTS's. On the other hand, our new findings of the extraordinary change of electronic correlation and quasiparticle scattering properties of dopants in FeHTS's provide an alternative and likely unifying view to understanding the complex phase diagrams of various series of FeHTS's and their unconventional superconductivity. For example, the anomalous impurity scattering behaviors could explain (at least partially) the different residual electrical resistivities [30], the robust superconductivity against heavy doping, and the different maximal $T_{C}$ 's and superconducting dome sizes in different series of FeHTS's.

We also show that the superconductivity could only emerge in a quite ubiquitous range of relative bandwidth. The increased bandwidth or suppressed electronic correlation by both heterovalent and isovalent dopants gives a natural explanation of their similar phase diagrams. Moreover, since the increased bandwidth is in harmony with the reduced Fe-anion bond length, our findings would help to bridge the gap between the structural parameters and superconductivity; that is, changing the lattice structure, such as the bond length, will significantly alter the electronic correlation and further affect the superconductivity. Finally, our results suggest that moderate electronic correlation plus minimal impurity scattering in the Fe-anion layer are the essential factors for maximizing $T_{C}$ in FeHTS's.

Therefore, many puzzling and seemingly random phenomena of FeHTS's could be comprehended (at least in the next step) after realizing these multifold roles of doping. In particular, our results indicate that electronic correlation is most likely the pivotal parameter for FeHTS's, rather than the Fermi surface, which should be expected but has unfortunately been ignored so far since the starting parent compound of FeHTS's is a metal instead of a Mott insulator for cuprate superconductors.

\section{EXPERIMENT}

Many FeHTS series were studied in this work, including two 111 series $\left[\mathrm{NaFe}_{1-x} \mathrm{Co}_{x} \mathrm{As}\right.$ (Ref. [13]) and 
$\mathrm{LiFe}_{1-x} \mathrm{Co}_{x} \mathrm{As}$ (Ref. [14])], three 122 series $\left[\mathrm{Ba}_{1-x} \mathrm{~K}_{x} \mathrm{Fe}_{2} \mathrm{As}_{2}\right.$ (Ref. [6]), $\mathrm{BaFe}_{2}\left(\mathrm{As}_{1-x} \mathrm{P}_{x}\right)_{2}$ (Ref. [9]), and $\mathrm{Ba}\left(\mathrm{Fe}_{1-x} \mathrm{Ru}_{x}\right)_{2} \mathrm{As}_{2}$ (Ref. [10])], one 11 series [ $\mathrm{Fe}_{1.04} \mathrm{Te}_{1-x} \mathrm{Se}_{x}$ (Ref. [31])], and $\mathrm{K}_{x} \mathrm{Fe}_{2-y} \mathrm{Se}_{2}$ (Ref. [32]), etc. For each series, high-quality single crystals of various dopings were synthesized according to the cited references, which also give corresponding phase diagrams. The samples are named by their dopant percentages throughout the paper. For example, the $x=0,0.03,0.09,0.12,0.17$, and 0.3 samples of $\mathrm{LiFe}_{1-x} \mathrm{Co}_{x}$ As are named LiFeAs, LC3, LC9, LC12, LC17, and LC30, respectively. ARPES measurements were performed at Fudan University with $21.2 \mathrm{eV}$ of light from a helium-discharging lamp, and also at various beamlines, including the beamline 5-4 of Stanford Synchrotron Radiation Lightsource (SSRL), the beamline 1 and beamline 9A of Hiroshima Synchrotron Radiation Center (HiSOR), and the SIS beamline of Swiss Light Source (SLS). All the data were taken with Scienta R4000 electron analyzers. The overall energy resolution was 5-10 meV at Fudan, SSRL, and HiSOR, or 15-20 meV at SLS depending on the photon energy; the angular resolution was 0.3 degrees. The samples were cleaved in situ and measured in ultrahigh vacuum with pressure better than $3 \times 10^{-11}$ torr.

\section{RESULTS}

\section{A. Quasiparticle scattering}

Figure 1(a) shows the doping evolution of the photoemission intensity distribution parallel to the $\Gamma-\mathrm{M}$ direction in $\mathrm{LiFe}_{1-x} \mathrm{Co}_{x} \mathrm{As}$. There are three holelike bands, $\alpha, \beta$, and $\gamma$, around the zone center and two electronlike bands, $\delta$ and $\eta$, around the zone corner. According to previous polarization-dependent ARPES results [29,33], the inner $\alpha$ and $\beta$ bands originate from the $d_{x z}$ and $d_{y z}$ orbitals, respectively, while the outer $\gamma$ band is constructed by the $d_{x y}$ orbital. The $\delta$ and $\eta$ electronlike bands are formed by the $d_{x z}, d_{y z}$, and $d_{x y}$ orbitals.

With Co doping, it is clear that the $d_{x y}$-based $\gamma$ band becomes significantly weaker and broader [Fig. 1(a)]. Figures 1(b)-1(e) plot the momentum distribution curves (MDCs) at the Fermi energy $\left(E_{F}\right)$ and $50 \mathrm{meV}$ below the band tops of the $\alpha$ and $\beta$ bands (so that the bands are resolvable), together with the full width at half maximum (FWHM) of each band, which reflects the scattering strength. The FWHM of $\gamma$ increases remarkably with Co doping. On the contrary, the FWHM of all the other bands does not change much with doping. We note that there are slight increases of the FWHMs with doping for the $\alpha$ and $\beta$ bands at $50 \mathrm{meV}$ below their band tops in Fig. 1(e). However, this is actually because the band tops of $\alpha$ and $\beta$ shift to higher binding energies with increasing electron density, which would enhance the scatterings according to Landau's Fermi liquid theory. Therefore, the quasiparticle lifetimes of $\eta, \alpha$, and $\beta$ are essentially insensitive to the
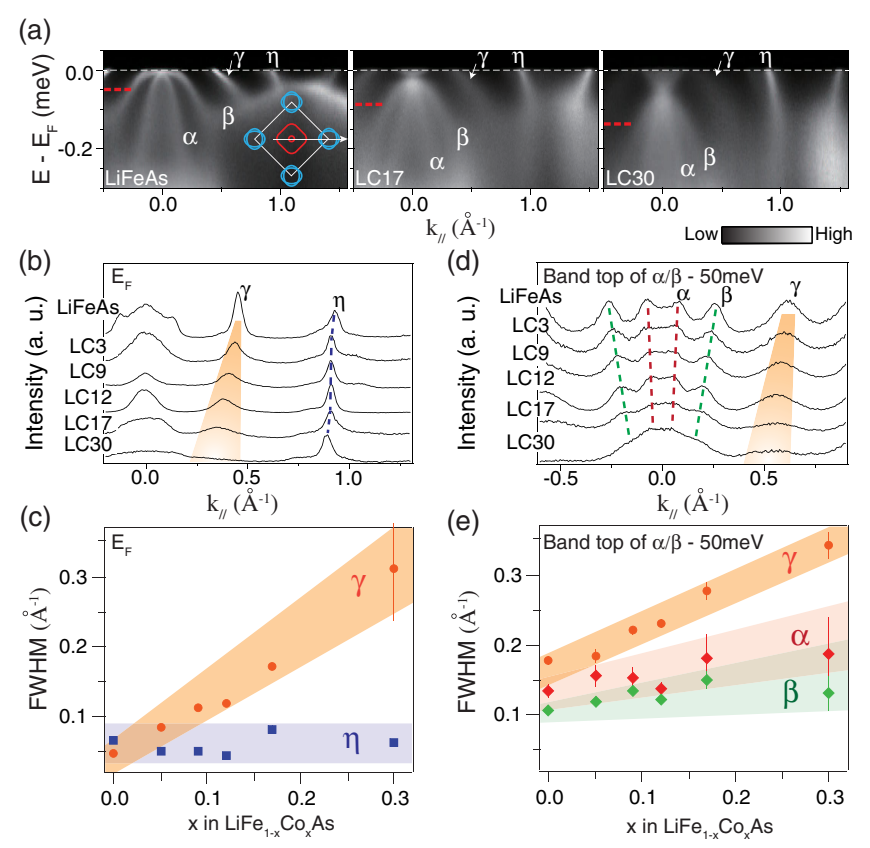

FIG. 1. (a) Doping dependence of the photoemission intensity distribution in $\mathrm{LiFe}_{1-x} \mathrm{Co}_{x}$ As parallel to the $\Gamma$-M direction, as indicated by the inset, taken with mixed polarized photons. The red dashed lines illustrate the energy positions of the MDCs in panel (d). (b) Doping dependence of the MDCs at $E_{F}$. Panel (c) shows the corresponding FWHMs of $\gamma$ and $\eta$ in panel (b). (d) MDCs at $50 \mathrm{meV}$ below the band tops of $\alpha$ and $\beta$ as a function of doping, since $\alpha$ and $\beta$ do not cross $E_{F}$. Panel (e) is the corresponding FWHMs of $\alpha, \beta$, and $\gamma$ in panel (d). The error bars of FWHM in panels (c) and (e) are standard deviations of the Lorentzian fit to MDC peaks.

increase of Co dopants, while the $\gamma$ band made of the $d_{x y}$ orbital is much more susceptible.

We have also observed similar spectra-broadening behavior for the $\gamma$ band in $\mathrm{NaFe}_{1-x} \mathrm{Co}_{x}$ As and $\mathrm{Fe}_{1.04} \mathrm{Te}_{1-x} \mathrm{Se}_{x}$ [Figs. 2(a)-2(d)]. However, in $\mathrm{Ba}_{1-x} \mathrm{~K}_{x} \mathrm{Fe}_{2} \mathrm{As}_{2}$, such a behavior appears to be absent [Figs. 2(e) and 2(f)]. Figure 2(g) compares the doping dependence of the MDC FWHM of the $\gamma$ band as a function of doping for various series of compounds. The spectra-broadening effect is strongly site dependent. The broadening of $\gamma$ is most pronounced in $\mathrm{NaFe}_{1-x} \mathrm{Co}_{x} \mathrm{As}$ and almost negligible in $\mathrm{Ba}_{1-x} \mathrm{~K}_{x} \mathrm{Fe}_{2} \mathrm{As}_{2}$.

The observation of such spectra broadening is not due to the increase of electronic correlations, as the band renormalization factor decreases with doping, which will be shown later in Fig. 4. It cannot be explained by the $k_{z}$ broadening effect in the photoemission process since the $\gamma$ band does not show pronounced $k_{z}$ dispersion [33]. The fact that the broadening is band dependent and could be observed in various compounds with different cleaved surfaces could also exclude the scattering effects of the sample surface. Therefore, the broadening of $\gamma$ is more likely an impurity scattering effect induced by the dopants, which naturally 
(a)

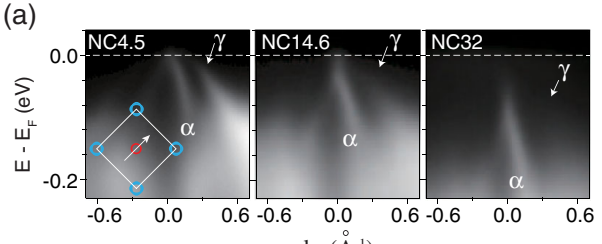

(c)

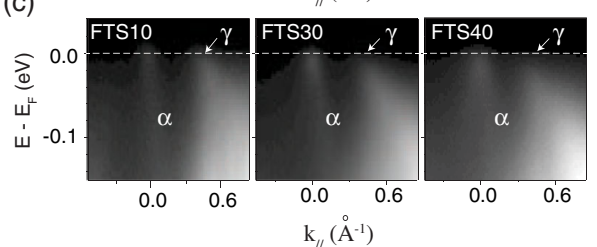

(e)

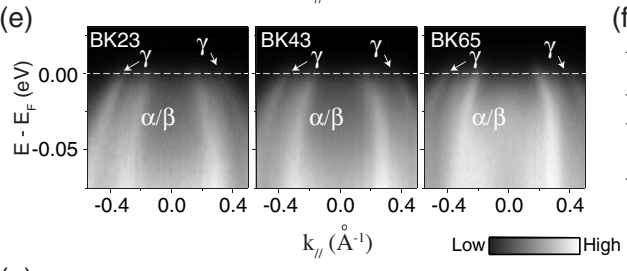

(g)

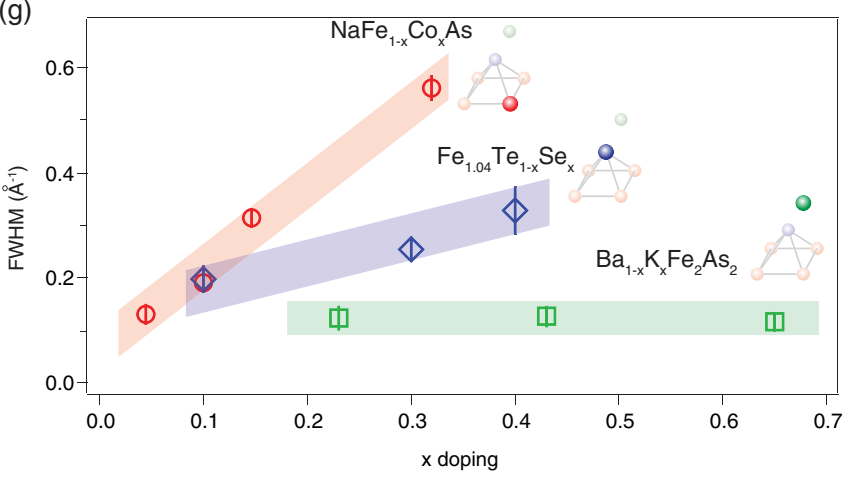

FIG. 2. (a) Doping dependence of the photoemission intensity distribution in $\mathrm{NaFe}_{1-x} \mathrm{Co}_{x}$ As parallel to the $\Gamma$-X direction, as indicated by the inset. (b) Doping dependence of the MDCs at $50 \mathrm{meV}$ below the band top of $\alpha$. Note that the increasing binding energy could only contribute a small increase to FWHM, which is less than $0.05 \AA^{-1}$, as shown in Fig. 1(e). Panels (c), (d), (e), and (f) are the same as (a) and (b), but for $\mathrm{Fe}_{1.04} \mathrm{Te}_{1-x} \mathrm{Se}_{x}$ and $\mathrm{Ba}_{1-x} \mathrm{~K}_{x} \mathrm{Fe}_{2} \mathrm{As}_{2}$, respectively. The MDCs in (d) and (f) were extracted from $E_{F}$. Note that the data for $\mathrm{NaFe}_{1-x} \mathrm{Co}_{x} \mathrm{As}$ and $\mathrm{Fe}_{1.04} \mathrm{Te}_{1-x} \mathrm{Se}_{x}$ were taken with $s$ polarized photons, and those for $\mathrm{Ba}_{1-x} \mathrm{~K}_{x} \mathrm{Fe}_{2} \mathrm{As}_{2}$ were taken with mixed polarized photons. Panel (g) summarizes the doping evolutions of the FWHMs of the $\gamma$ bands in $\mathrm{NaFe}_{1-x} \mathrm{Co}_{x} \mathrm{As}, \mathrm{Fe}_{1.04} \mathrm{Te}_{1-x} \mathrm{Se}_{x}$ and $\mathrm{Ba}_{1-x} \mathrm{~K}_{x} \mathrm{Fe}_{2} \mathrm{As}_{2}$ series. The error bars of FWHMs in panel (g) are standard deviations of the Lorentzian fit to MDC peaks.

explains the site dependence observed here: When the dopant moves away from the Fe-anion layer, the scattering strength gradually decreases.

\section{B. Alteration of electron correlation}

Besides scattering the quasiparticles, dopants also affect the electronic structure by changing the electronic correlation. The band renormalization factor, a ratio between the calculated bandwidth from density functional theory and the bandwidth observed by ARPES, can be regarded as a measure of the electronic correlation strength. Since the calculated bandwidth usually shows weak doping dependence [34-37], the increase of the bandwidth with doping observed by ARPES indicates a decrease of the electronic correlation strength.

In $\mathrm{BaFe}_{2}\left(\mathrm{As}_{1-x} \mathrm{P}_{x}\right)_{2}$, the bandwidth and Fermi velocity were found to increase significantly with $\mathrm{P}$ doping, indicating the decrease of electronic correlations under compressional chemical pressure [9]. In Fig. 3, we show that such a phenomenon can be rather ubiquitously observed in Co-, Se-, and Ru-doped FeHTS's. For example, as shown in Fig. 3(a), the band structure measured from $\mathrm{LiFeAs}$ could match the bands in LC17 or LC30 well, after it is shifted in energy and scaled by a factor of around 1.6 or 2.2 , respectively. This shows that the bandwidth increases equally for all the bands. Since the top and bottom of the $\beta$ band can both be observed in most cases, we take the bandwidth of $\beta$ as a characterization of the overall Fe $3 d$ bandwidth [Fig. 3(b)].

The same analysis on the evolution of the $\beta$ bandwidth with doping was extended to $\mathrm{NaFe}_{1-x} \mathrm{Co}_{x} \mathrm{As}$, $\mathrm{Ba}_{1-x} \mathrm{~K}_{x} \mathrm{Fe}_{2} \mathrm{As}_{2}, \quad \mathrm{BaFe}_{2}\left(\mathrm{As}_{1-x} \mathrm{P}_{x}\right)_{2}, \mathrm{Fe}_{1.04} \mathrm{Te}_{1-x} \mathrm{Se}_{x}$, and $\mathrm{Ba}\left(\mathrm{Fe}_{1-x} \mathrm{Ru}_{x}\right)_{2} \mathrm{As}_{2}$, as shown in Figs. 3(c)-3(1). Note that, while the band top of $\beta$ is below $E_{F}$ in $\mathrm{LiFe}_{1-x} \mathrm{Co}_{x} \mathrm{As}$ and $\mathrm{NaFe}_{1-x} \mathrm{Co}_{x}$ As [Figs. 3(a) and 3(c)] or just touches $E_{F}$ in $\mathrm{Fe}_{1.04} \mathrm{Te}_{1-x} \mathrm{Se}_{x}$ [Fig. 3(i)], the $\beta$ bands in $\mathrm{Ba}_{1-x} \mathrm{~K}_{x} \mathrm{Fe}_{2} \mathrm{As}_{2}$ [Fig. 3(e)], $\mathrm{BaFe}_{2}\left(\mathrm{As}_{1-x} \mathrm{P}_{x}\right)_{2}$ [Fig. 3(g)], and $\mathrm{Ba}\left(\mathrm{Fe}_{1-x} \mathrm{Ru}_{x}\right)_{2} \mathrm{As}_{2}$ [Fig. 3(k)] cross $E_{F}$ near the zone center. In order to determine the energy position of the band top of $\beta$ in $\mathrm{Ba}_{1-x} \mathrm{~K}_{x} \mathrm{Fe}_{2} \mathrm{As}_{2}, \mathrm{BaFe}_{2}\left(\mathrm{As}_{1-x} \mathrm{P}_{x}\right)_{2}$, and $\mathrm{Ba}\left(\mathrm{Fe}_{1-x} \mathrm{Ru}_{x}\right)_{2} \mathrm{As}_{2}$, we applied a parabolic-curve fitting to the $\beta$ band dispersion for every doping in each series. The fitted curves follow the band dispersions of $\beta$ below $E_{F}$ well, and the fitted effective mass of $\beta$ near the zone center shows consistent doping dependence with the bandwidth in all three systems. We further quantitatively summarize the doping dependence of the bandwidths in various systems, as shown in Fig. 4. The increase of the bandwidth with doping is ubiquitous in all the studied systems except for $\mathrm{Ba}_{1-x} \mathrm{~K}_{x} \mathrm{Fe}_{2} \mathrm{As}_{2}$ [Fig. 4(c)], where the bandwidth of $\beta$ shows a very small decrease or is almost insensitive to the $\mathrm{K}$ doping after considering the error bars. We will discuss the possible causes in detail in Sec. IV B.

\section{DISCUSSIONS}

As we have shown above, besides the well-known Fermi-surface alteration, the dopants could significantly change the electronic structure in two other ways: (1) by scattering the quasiparticles of the central $d_{x y}$-based $\gamma$ band, whose strength strongly depends on the site of dopants, and (2) by suppressing the electronic correlation for various systems except for $\mathrm{Ba}_{1-x} \mathrm{~K}_{x} \mathrm{Fe}_{2} \mathrm{As}_{2}$. In parts $\mathrm{A}$ and $\mathrm{B}$ of this section, we will discuss the implications of our findings, particularly on the superconductivity. On this basis, we will 

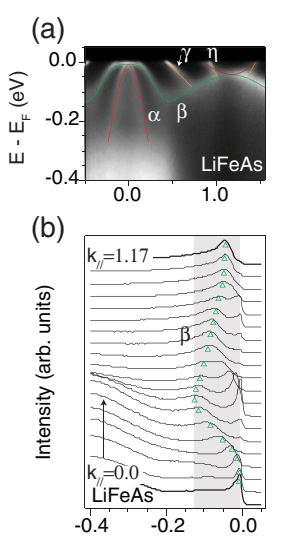

(e)
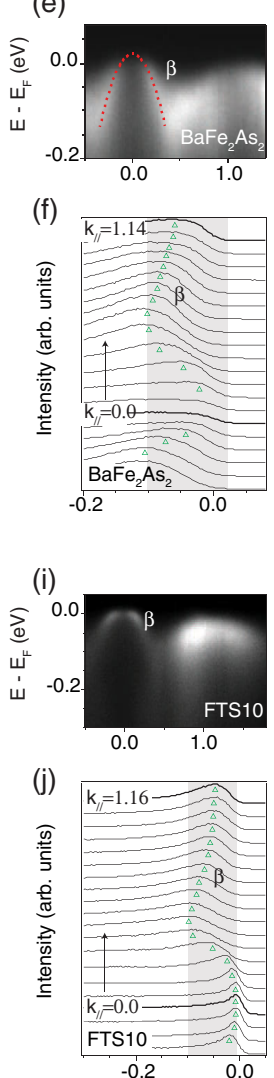

$\mathrm{LiFe}_{1-x} \mathrm{Co}_{x} \mathrm{As}$
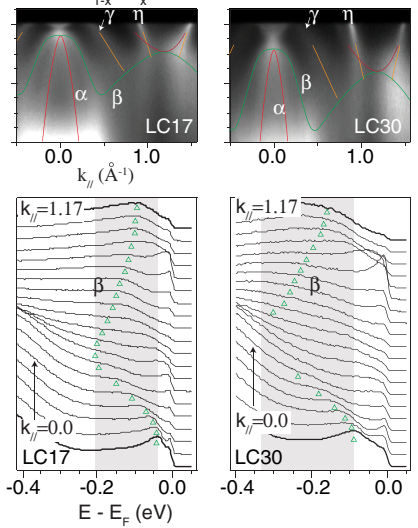

$\mathrm{Ba}_{1-\mathrm{K}} \mathrm{K}_{\mathrm{x}} \mathrm{Fe}_{2} \mathrm{As}_{2}$
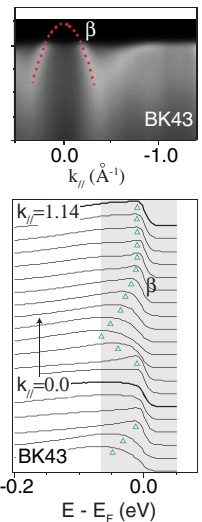

$\mathrm{Fe}_{1.04} \mathrm{Te}_{1-\mathrm{x}} \mathrm{Se}_{\mathrm{x}}$

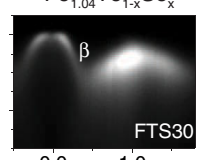

$0.0 \mathrm{k}_{/ /}\left(\AA^{-1}\right){ }^{-1.0}$
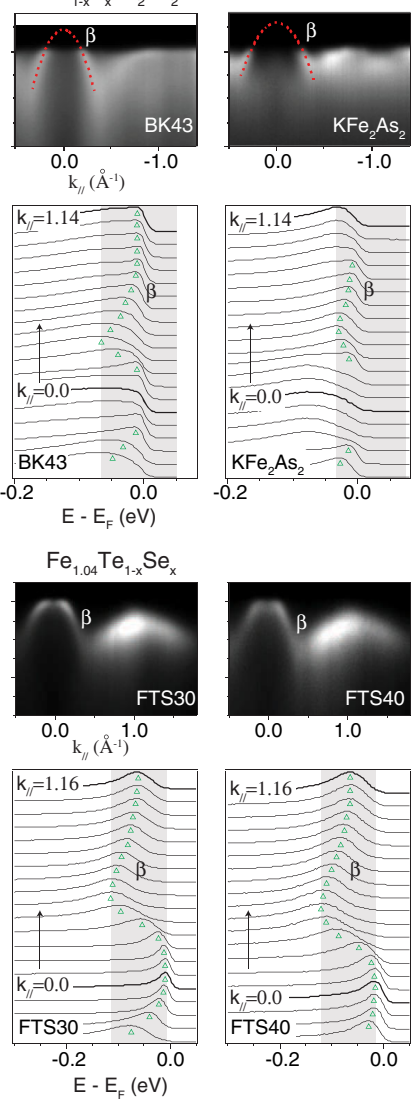

(c)

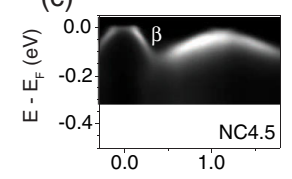

(d)
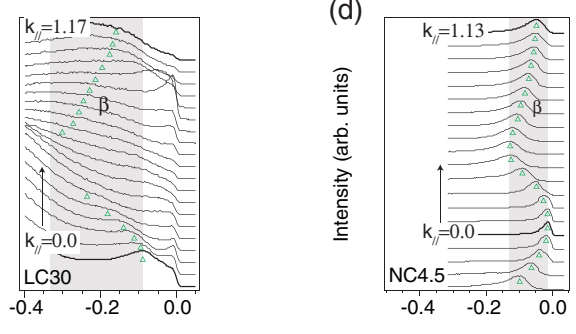

(g)

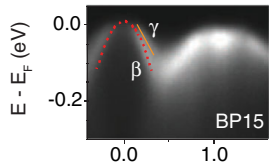

(h)

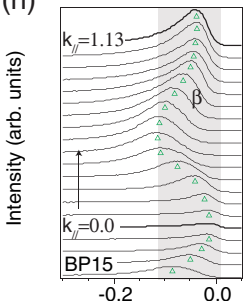

(k)

(I)
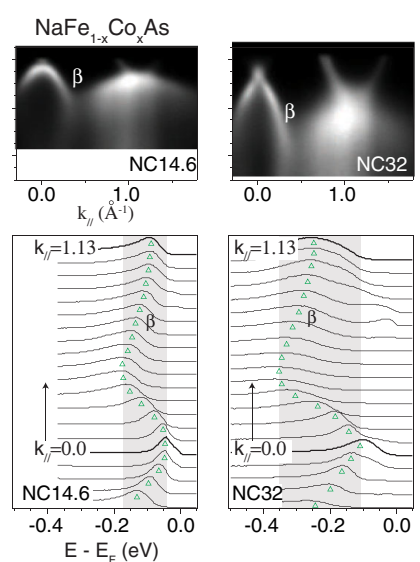

$\mathrm{BaFe}_{2}\left(\mathrm{As}_{1-x} \mathrm{P}_{x}\right)_{2}$
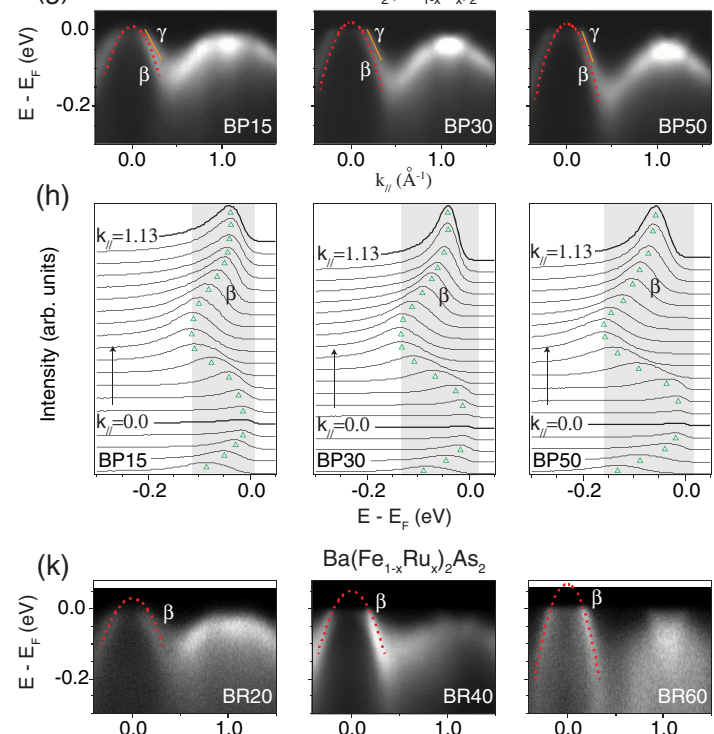

$\mathrm{Ba}\left(\mathrm{Fe}_{1-\mathrm{x}} \mathrm{Ru}_{\mathrm{x}}\right)_{2} \mathrm{As}_{2}$
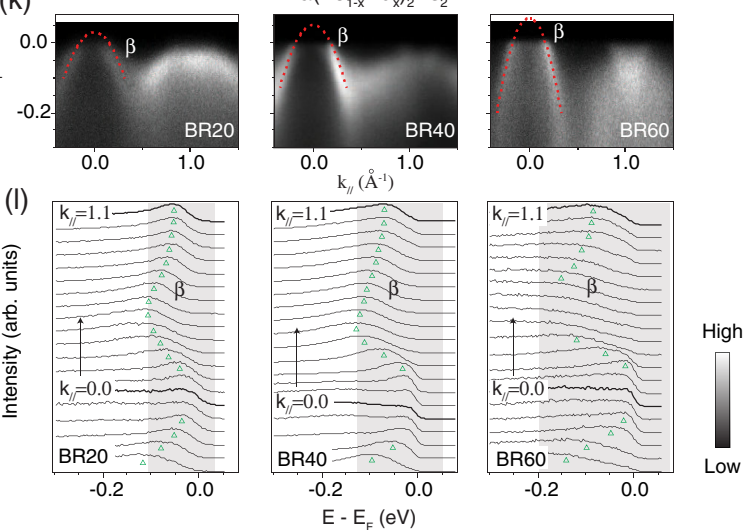

FIG. 3. (a) Doping dependence of the photoemission intensity distributions parallel to the $\Gamma-\mathrm{M}$ direction in $\mathrm{LiFe}_{1-x} \mathrm{Co}_{x} \mathrm{As}$, taken with mixed polarized photons. The band structure was determined based on the photoemission data in LiFeAs. The entire band structure was shifted in energy and normalized by a factor of around 1.6 in LC17 and around 2.2 in LC30. The obtained band structures were overlaid on the photoemission data in LC17 and LC30. (b) Corresponding energy distribution curves (EDCs) of the data in panel (a). The intensities of the EDCs were normalized to enhance the $\beta$ band. The green triangles trace the band dispersion of $\beta$. The gray shaded area is a guide to the eyes for viewing the change of the bandwidth with doping. (c), (e), (g), (i), and (k) Doping dependence of the photoemission intensity distributions taken parallel to the $\Gamma$-M direction with $s$ polarized photons in $\mathrm{NaFe}_{1-x} \mathrm{Co}_{x} \mathrm{As}, \mathrm{Ba}_{1-x} \mathrm{~K}_{x} \mathrm{Fe}_{2} \mathrm{As}_{2}$, $\mathrm{BaFe}_{2}\left(\mathrm{As}_{1-x} \mathrm{P}_{x}\right), \mathrm{Fe}_{1.04} \mathrm{Te}_{1-x} \mathrm{Se}_{x}$, and $\mathrm{Ba}\left(\mathrm{Fe}_{1-x} \mathrm{Ru}_{x}\right)_{2} \mathrm{As}_{2}$, respectively. The photoemission data for each series are from the same $k_{z}$, although the bandwidth varies little with $k_{z}$ as reported before [9]. Note that the red dashed lines overlaid on panels (e), (g), and (k) are the quadratic-curve-fitting results for the $\beta$ bands, in order to determine the energy positions of $\beta$ band tops. Panels (d), (f), (h), (j), and (l) are the same as (b), but for $\mathrm{NaFe}_{1-x} \mathrm{Co}_{x} \mathrm{As}, \mathrm{Ba}_{1-x} \mathrm{~K}_{x} \mathrm{Fe}_{2} \mathrm{As}_{2}, \mathrm{BaFe}_{2}\left(\mathrm{As}_{1-x} \mathrm{P}_{x}\right), \mathrm{Fe}_{1.04} \mathrm{Te}_{1-x} \mathrm{Se}_{x}$, and $\left.\mathrm{Ba} \mathrm{Fe}_{1-x} \mathrm{Ru}_{x}\right)_{2} \mathrm{As}_{2}$, respectively. 
(a)

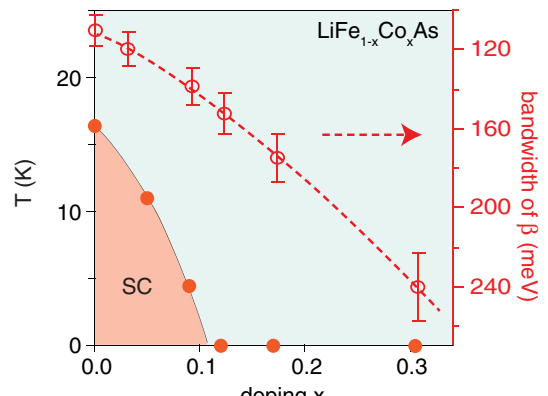

(d)

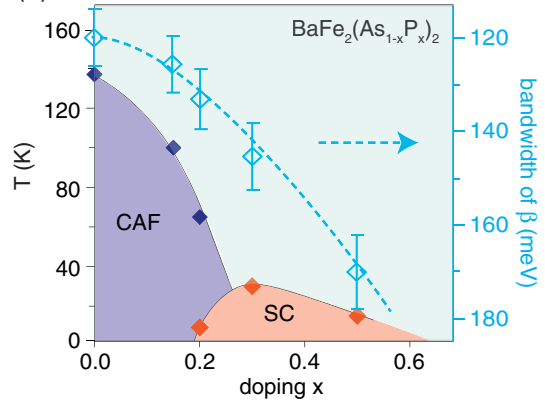

(b)

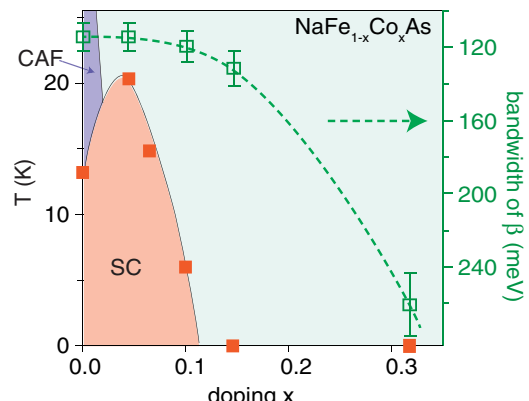

(e)

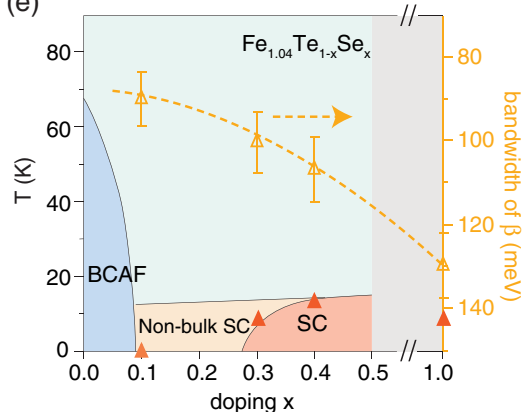

(c)

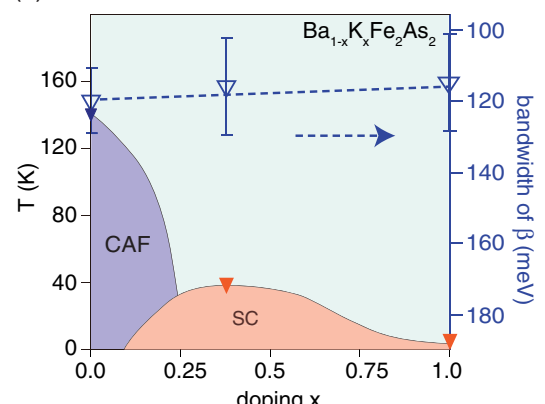

(f)

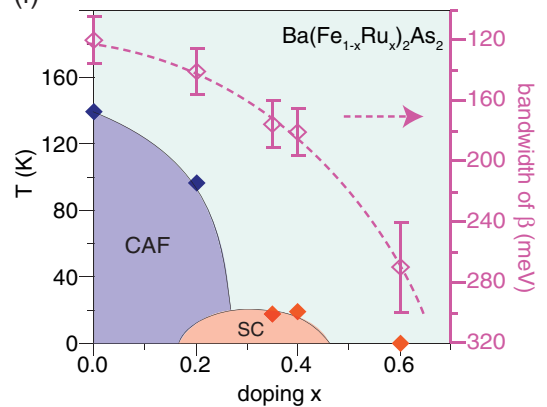

FIG. 4. (a) Evolutions of $T_{C}$ and $\beta$ bandwidths with doping in $\mathrm{LiFe}_{1-x} \mathrm{Co}_{x}$ As. Panels (b)-(f) are the same as (a), but for $\mathrm{NaFe}_{1-x} \mathrm{Co}_{x} \mathrm{As}, \mathrm{Ba}_{1-x} \mathrm{~K}_{x} \mathrm{Fe}_{2} \mathrm{As}_{2}, \mathrm{BaFe}_{2}\left(\mathrm{As}_{1-x} \mathrm{P}_{x}\right)_{2}, \mathrm{Fe}_{1.04} \mathrm{Te}_{1-x} \mathrm{Se}_{x}$, and $\mathrm{Ba}\left(\mathrm{Fe}_{1-x} \mathrm{Ru}_{x}\right)_{2} \mathrm{As}_{2}$, respectively. The superconducting, collinear antiferromagnetic, and bicollinear antiferromagnetic phases are abbreviated as SC, CAF, and BCAF, respectively. The bandwidth for $\mathrm{Fe}_{1.04} \mathrm{Se}$ in panel (e) was extracted from Refs. [38,39]. Note that the doping range for $0.5<x<1.0$ in $\mathrm{Fe}_{1.04} \mathrm{Te}_{1-x} \mathrm{Se}_{x}$ cannot be chemically synthesized [40]. The phase diagrams for $\mathrm{Ba}_{1-x} \mathrm{~K}_{x} \mathrm{Fe}_{2} \mathrm{As}_{2}, \mathrm{BaFe}_{2}\left(\mathrm{As}_{1-x} \mathrm{P}_{x}\right)_{2}, \mathrm{Fe}_{1.04} \mathrm{Te}_{1-x} \mathrm{Se}_{x}$, and $\mathrm{Ba}\left(\mathrm{Fe}_{1-x} \mathrm{Ru}_{x}\right)_{2} \mathrm{As}_{2}$ were extracted from Refs. [9,10,12,41]. The error bars of $\beta$ bandwidths come from the uncertainty in the dispersion determination.

revisit the role of the Fermi surface on superconductivity by providing further evidence in part $\mathrm{C}$.

\section{A. Band-selective and site-dependent impurity scattering effect}

The dopants could significantly scatter the quasiparticles of the $d_{x y}$-originated $\gamma$ band around the zone center, while other bands are relatively unaffected. The scattering strength is the strongest when the dopant is in the Feanion layer. Such a band-selective and site-dependent impurity scattering effect needs further theoretical understanding. Nevertheless, our findings could explain many existing observations: (1) The superconductivity is robust against heavy doping in FeHTS's since most bands are basically unaffected by the scattering of dopants. (2) They could partially explain why the maximal $T_{C}$ 's of $\mathrm{NaFe}_{1-x} \mathrm{Co}_{x} \mathrm{As}$ and $\mathrm{Fe}_{1.04} \mathrm{Te}_{1-x} \mathrm{Se}_{x}$ are lower than that of $\mathrm{Ba}_{1-x} \mathrm{~K}_{x} \mathrm{Fe}_{2} \mathrm{As}_{2}$ because the quasiparticle near $E_{F}$ is strongly suppressed for the large $\gamma$ Fermi pocket in $\mathrm{NaFe}_{1-x} \mathrm{Co}_{x} \mathrm{As}$ and $\mathrm{Fe}_{1.04} \mathrm{Te}_{1-x} \mathrm{Se}_{x}$ [Figs. 2(a)-2(d)], which thus likely does not contribute to the superconductivity [42]. Similar to $\mathrm{Ba}_{1-x} \mathrm{~K}_{x} \mathrm{Fe}_{2} \mathrm{As}_{2}$, the superconductivity in the so-called 1111 series is obtained by doping off the Feanion plane as well [43]. The record high $T_{C}$ of $56 \mathrm{~K}$ in this series may be related to the weak scattering of the off-plane dopants. (3) They may partially explain that the doping range for the superconducting dome increases in the general order of compounds with Co dopants (typically very narrow), those with $\mathrm{P}$ or $\mathrm{Se}$ dopants (typically covering a third to half of the phase diagram), and those with off-plane $\mathrm{K}$ or $\mathrm{F}$ dopants (typically covering more than half of the phase diagram). The recently discovered phase diagram of $\mathrm{LaFeAsO}_{1-x} \mathrm{H}_{x}$ exhibits a large superconducting dome with a rather flat top, where its $T_{C}$ is independent of the doping [16]. This shows that the impurity scattering strength caused by hydrogen $(\mathrm{H})$, which is off the FeAs plane, should be weak in $\mathrm{LaFeAsO}_{1-x} \mathrm{H}_{x}$ as well, and the superconductivity may be insensitive to carrier density variation over a large range. (4) They provide a microscopic understanding of why the residual electrical resistivity decreases in the order of Co-doped, P-doped, and K-doped $\mathrm{BaFe}_{2} \mathrm{As}_{2}$ reported recently by Ref. [30]. (5) A recent STM study on $\mathrm{NaFe}_{1-x} \mathrm{Co}_{x}$ As shows that the low-energy electronic state is somehow insensitive to the Co dopants [44]. Our results provide an explanation: The tunneling matrix element is dominated by the $d_{x z} / d_{y z}$ states, which extend out of the plane and are inert to impurity scattering, rather than the $\gamma$ band made of the in-plane $d_{x y}$ orbital.

\section{B. Origin and critical role of electronic correlation}

For a correlated material, the strength of the electronic correlation is a critical parameter to determine its 
properties. In the Hubbard model for describing the Mott (insulator-to-metal) transition, the electronic correlation strength is determined by the ratio between the on-site Coulomb repulsion and the electron kinetic energy. The suppression of the electronic correlation can be achieved in two different ways: (1) by increasing carriers with doping, which could lead to better screening effect and thus smaller effective Coulomb interactions between electrons, and (2) by shrinking the bond length, which could increase the electron hopping and thus enlarge the electron kinetic energy. These are the so-called filling-control and bandwidth-control methods, respectively.

For the FeHTS's discussed here, the electronic correlation is almost doping independent for the hole-doped compound $\mathrm{Ba}_{1-x} \mathrm{~K}_{x} \mathrm{Fe}_{2} \mathrm{As}_{2}$ [Fig. 4(c)], while for $\mathrm{BaFe}_{2}\left(\mathrm{As}_{1-x} \mathrm{P}_{x}\right)_{2}$ and $\mathrm{Fe}_{1.04} \mathrm{Te}_{1-x} \mathrm{Se}_{x}$, the carrier density is almost unchanged but the electronic correlation decreases significantly [Figs. 4(d) and 4(e)]. As shown in Fig. 5(a), the bond length of Fe-As or Fe-Te decreases with the doping in $\mathrm{LiFe}_{1-x} \mathrm{Co}_{x} \mathrm{As}, \mathrm{NaFe}_{1-x} \mathrm{Co}_{x} \mathrm{As}$, $\mathrm{BaFe}_{2}\left(\mathrm{As}_{1-x} \mathrm{P}_{x}\right)_{2}$, and $\mathrm{Fe}_{1.04} \mathrm{Te}_{1-x} \mathrm{Se}_{x}$, because of the smaller ionic radii of the $\mathrm{Co}, \mathrm{P}$, and Se dopants, than those of elements substituted by them. In contrast, the $\mathrm{K}$ dopants in $\mathrm{Ba}_{1-x} \mathrm{~K}_{x} \mathrm{Fe}_{2} \mathrm{As}_{2}$ are out of the FeAs plane, and the bond length is thus unchanged with doping. In Fig. 5(b), we plot the evolution of the $\beta$ bandwidth with the Fe-As or Fe-Te bond length in each series. One finds that the bandwidth of $\beta$ increases with the decrease of the bond length. Therefore, the electronic correlation in FeHTS's is closely related to the structure parameters, such as the bond length we found here. $\mathrm{Ba}\left(\mathrm{Fe}_{1-x} \mathrm{Ru}_{x}\right)_{2} \mathrm{As}_{2}$ seems to be an exception since its bond length increases slightly with doping [45]. However, the large orbital radius of the $\mathrm{Ru} 4 d$ electron, which could also enhance the electron hopping, may overcome the enlarged bond length and thus result in the decrease of the electronic correlation [Fig. 4(f)].

Intriguingly, the bond length shrinks at a similar rate with doping for the Co and P/Se dopants, as shown in Fig. 5(a). However, the bandwidth increases much more significantly for Co-doped compounds [Fig. 5(c)]. Such an additional suppression of electronic correlation could be attributed to the enhanced screening effect induced by more carriers. Following this scenario, it is difficult to understand the fact that the electronic correlation is not suppressed but rather slightly enhanced in heavily hole-doped $\mathrm{Ba}_{1-x} \mathrm{~K}_{x} \mathrm{Fe}_{2} \mathrm{As}_{2}$ [Fig. 4(c)]. To understand this dilemma, one has to realize that the parent compound of FeHTS is not a half-filled Mott insulator. For a half-filling band system where electronic correlations originate from intraband Coulomb interactions, such as cuprates, both hole and electron doping suppress the electronic correlation. The phase diagram is particle-hole symmetric. FeHTS is a Fe $3 d^{6}$ multiband system; as a result, it has been proposed that the electronic correlations are mainly due to the Hund's rule coupling $J_{H}$ instead of the intraband Coulomb interaction $[48,49]$. In (a)

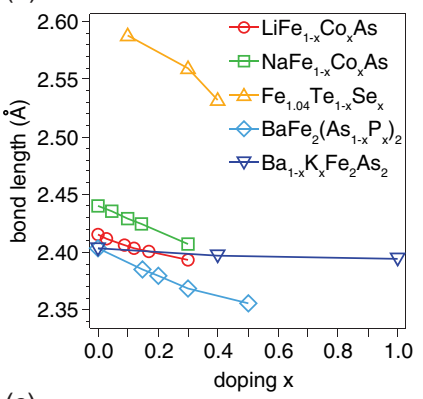

(b)
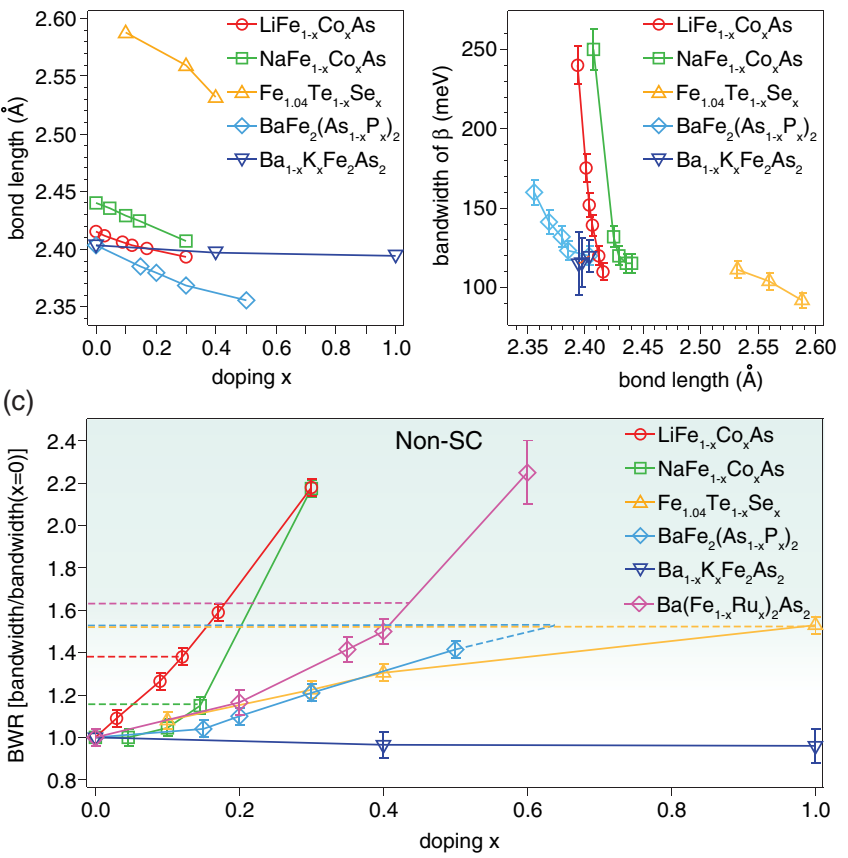

FIG. 5. (a) Evolutions of the Fe-As bond length in $\mathrm{LiFe}_{1-x} \mathrm{Co}_{x} \mathrm{As}, \quad \mathrm{NaFe}_{1-x} \mathrm{Co}_{x} \mathrm{As}, \quad \mathrm{BaFe}_{2}\left(\mathrm{As}_{1-x} \mathrm{P}_{x}\right)_{2}$, and $\mathrm{Ba}_{1-x} \mathrm{~K}_{x} \mathrm{Fe}_{2} \mathrm{As}_{2}$, or the Fe-Te bond length in $\mathrm{Fe}_{1.04} \mathrm{Te}_{1-x} \mathrm{Se}_{x}$. The bond length data were extracted from Ref. [46]. Note that, in the case where the bond length data for certain doping-level samples are lacking, we estimate the bond length values by the linear interpolation method. Panel (b) summarizes the evolutions of the $\beta$ bandwidths as a function of the Fe-As or Fe-Te bond length in these five series. (c) Doping dependence of the $\beta$ bandwidth normalized by that of its parent compound $(x=0)$, named BWR (bandwidth ratio) for simplicity, in each series. The horizontal dashed line divides the superconducting region and overdoped nonsuperconducting region of each series. Note that the bandwidth of FeTe, the parent compound of $\mathrm{Fe}_{1.04} \mathrm{Te}_{1-x} \mathrm{Se}_{x}$, was estimated from a linear extrapolation of data in Fig. 4(e) since the photoemission data of FeTe is intrinsically very broad [47], making it difficult to directly determine its bandwidth. The error bars of $\beta$ bandwidths or normalized bandwidths in panels (b) and (c) come from the uncertainty in the dispersion determination.

this case, the hole doping actually drives the system towards the $3 d^{5}$ state where the strength of Hund's interaction is strongest, which is likely counterbalanced by the screening effects, giving the observed dopingindependent electronic correlation. On the other hand, the electron doping drives the system towards the $3 d^{7}$ state and further reduces the electronic correlation. Therefore, the particle-hole asymmetric electronic correlation observed here could be viewed as positive evidence for the importance of Hund's rule coupling in inducing the electronic correlations in FeHTS's.

From the above discussions, one could thus conclude that the change of the electronic correlation in FeHTS's originates from two aspects: (1) The carrier doping significantly affects the electronic interactions, such as Hund's 
interaction, and alters the electronic correlation in a particle-hole asymmetric fashion, and (2) the change of lattice structure, such as bond length, could suppress the electronic correlation by increasing the kinetic energy of electrons. One may find that such behaviors have some resemblance to the effects of the filling-control and bandwidth-control methods in Mott physics. However, since the parent compound of FeHTS's is a multiband metal instead of a single-band Mott insulator, there are clearly fundamental differences in the nature of correlations and how they react to doping.

Furthermore, it was proposed that the superconductivity in FeHTS's could be mediated by spin or orbital fluctuations [26,50-52], while the strength of such fluctuations is related to the electronic correlations. In other words, when the electronic correlation is weak, spin or orbital fluctuations will not be sufficient to mediate the superconducting pairing. Consistently, our data show that the system becomes nonsuperconducting when the observed bandwidth is sufficiently large. Taking the end members of $\mathrm{BaFe}_{2} \mathrm{P}_{2}$ and $\mathrm{LaOFeP}$ as examples, the quantum fluctuations in these two compounds are strongly suppressed by $\mathrm{P}$ dopants, and the two systems were reported to behave more like normal metals with rather weak electronic correlations $[53,54]$. Quantitatively, we observed that the boundary in the BWR (bandwidth ratio between the bandwidth and that of its parent compound) between the superconducting region and the nonsuperconducting region is between 1.2 and 1.6 [Fig. 5(c)], depending on the series. In general, Co-doped series, $\mathrm{LiFe}_{1-x} \mathrm{Co}_{x}$ As and $\mathrm{NaFe}_{1-x} \mathrm{Co}_{x}$ As here, have smaller boundary BWR values or narrower superconducting regions, which might be caused by the stronger impurity scatterings there. Overall, superconductivity cannot be sustained for compounds with BWR above about 1.5. On the other hand, when the electronic correlation is too strong, the system is in the magnetic or orbital ordered phase [Figs. 4(b)-4(e)], and the competing order would suppress superconductivity. For example, in FeTe, the normal state shows semiconductor behavior, and the magnetic moment is as large as $2 \mu_{B}$ in the low-temperature magnetic ordered states $[55,56]$.

Our results show that the superconductivity in FeHTS's is located at the moderate electronic correlation region. Too-strong or too-weak electronic correlations will not benefit from the emergence of superconductivity. This applies for all the FeHTS's studied here, no matter if the electronic correlation is suppressed by decreasing the bond length, carrier doping, or doping $4 d$ electrons. Therefore, the observed bandwidth or electronic correlation seems to be a more robust and relevant parameter than the carrier doping or the structural parameters, which thus provides a new view to understand similar phase diagrams obtained by the diversified ways of doping. However, we note that the specific value of the superconducting $T_{C}$ is not controlled only by the electronic correlation. $T_{C}$ is very sensitive to various factors, such as the impurity scattering strength that we have discussed extensively in part A.

\section{The secondary role of Fermi-surface topology on superconductivity}

Tuning the Fermi surface is another doping effect in FeHTS's which has been widely studied before. Previous ARPES results on $\mathrm{Ba}\left(\mathrm{Fe}_{1-x} \mathrm{Co}_{x}\right)_{2} \mathrm{As}_{2}$ and $\mathrm{NaFe}_{1-x} \mathrm{Co}_{x} \mathrm{As}$ have observed a correlation between the vanishing superconductivity and the Lifshitz transition of the center hole pockets [17,57], which suggested the critical role of the central hole pockets on the superconductivity. Here, in $\mathrm{LiFe}_{1-x} \mathrm{Co}_{x} \mathrm{As}$, we also found that, with Co doping, the band tops of the center $\alpha$ and $\beta$ hole bands shift downwards below $E_{F}$, and an electron band $\kappa$ could be observed in LC12 [Fig. 6(b)]. Meanwhile, superconductivity diminishes around LC12 in the phase diagram [Fig. 6(a)]. We note that, although the $d_{x y}$-based $\gamma$ hole pocket is present even in the heavily doped compounds LC12 and LC17, the quasiparticle of $\gamma$ near $E_{F}$ is ill defined because of the strong impurity scattering and cannot contribute to any superconducting pairing. Therefore, the Lifshitz transition observed here in $\mathrm{LiFe}_{1-x} \mathrm{Co}_{x} \mathrm{As}$, together with the previous observations in $\mathrm{Ba}\left(\mathrm{Fe}_{1-x} \mathrm{Co}_{x}\right)_{2} \mathrm{As}_{2}$ and $\mathrm{NaFe}_{1-x} \mathrm{Co}_{x} \mathrm{As}$, seems to be consistent with the possible crucial role of the interpocket scattering between the central hole pockets and the corner electron pockets on the superconductivity [50,51].

However, such a picture has been seriously challenged by the recent studies on $\mathrm{K}_{x} \mathrm{Fe}_{2-y} \mathrm{Se}_{2}$ and the monolayer FeSe thin film on a $\mathrm{SrTiO}_{3}(\mathrm{STO})$ substrate, where the $T_{C}$ 's are above $30 \mathrm{~K}$, but the Fermi surfaces are composed of

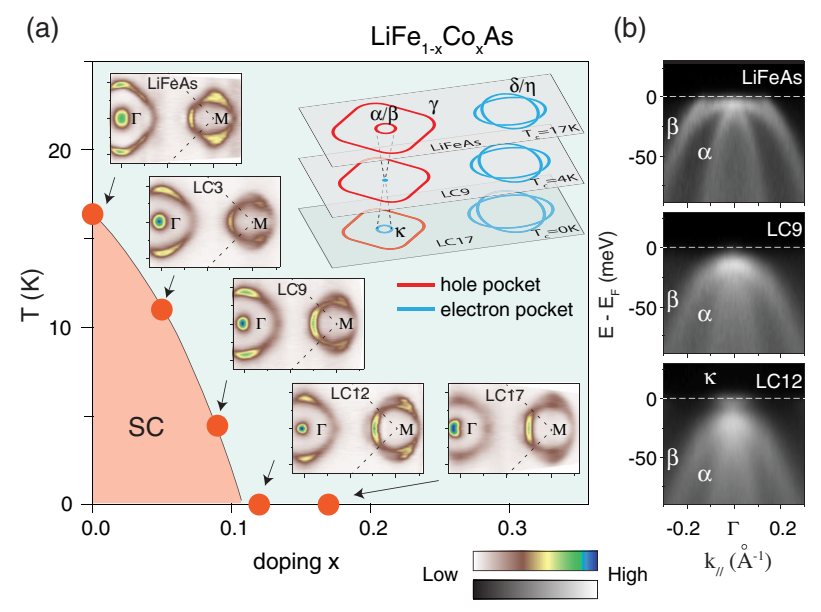

FIG. 6. (a) The phase diagram and corresponding photoemission intensity maps for $\mathrm{LiFe}_{1-x} \mathrm{Co}_{x}$ As. The top-right inset panel illustrates the doping dependence of the Fermi-surface topologies, where the hole and electron pockets are plotted with the red and blue lines, respectively. (b) Doping dependence of the photoemission intensity distributions taken near the zone center along the $\Gamma-\mathrm{M}$ direction for $\mathrm{LiFe}_{1-x} \mathrm{Co}_{x} \mathrm{As}$. 
only electron pockets without any central hole pocket [19-21]. One explanation is that the superconducting mechanisms of these iron selenides are remarkably different from the other FeHTS's. Other factors should be considered, for example, the phase separation between superconducting and insulating phases in $\mathrm{K}_{x} \mathrm{Fe}_{2-y} \mathrm{Se}_{2}$ (Refs. [58,59]) and the critical role of the substrate and interface in ultrathin FeSe film [60,61]. On the other hand, if the superconductivities in iron pnictides and iron selenides share a unified mechanism, the correlation between the Lifshitz transition and superconductivity observed in the Co-doped systems could be accidental. This is because, with the increasing Co concentration, not only is the Fermi-surface topology changed, but the electronic correlation also decreases at the same time [Figs. 4(a) and 4(b)], which could strongly suppress the pairing strength for superconductivity.

It is thus intriguing to compare the electronic structure of NC32 with that of $\mathrm{K}_{0.77} \mathrm{Fe}_{1.65} \mathrm{Se}_{2}$ since $\mathrm{NC} 32$ and $\mathrm{K}_{0.77} \mathrm{Fe}_{1.65} \mathrm{Se}_{2}$ possess similar Fermi-surface topology and size, but one is nonsuperconducting and the other has a $T_{C}$ above $30 \mathrm{~K}$ [Figs. 7(a) and 7(c)]. Figures 7(b) and $7(\mathrm{~d})$ compare their low-lying band structures. The difference is obvious. The larger bandwidth of the $\beta$ band and the smaller effective mass of the $\delta / \eta$ electron band in $\mathrm{NC} 32$ (than those in $\mathrm{K}_{0.77} \mathrm{Fe}_{1.65} \mathrm{Se}_{2}$ ) all indicate that the electronic correlation in NC32 is much weaker than that of
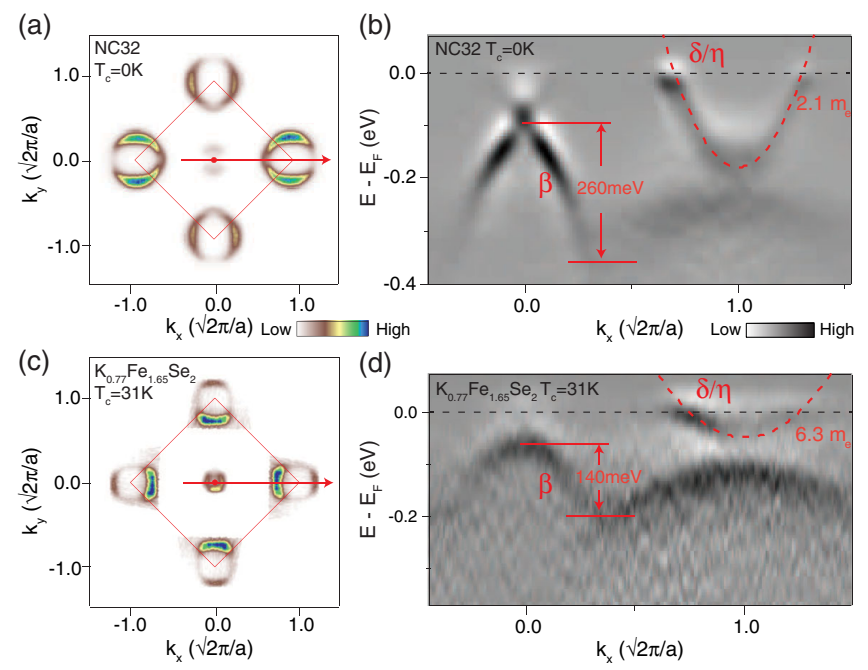

FIG. 7. (a) Photoemission intensity map across the $Z$ point for NC32, taken with $100-\mathrm{eV}$ photons in $s$ polarization. (b) The photoemission intensity distribution along the $Z$ - $A$ direction as illustrated by the red arrow in panel (a) for NC32, taken with $100-\mathrm{eV}$ photons in $s$ polarization. (c) Photoemission intensity map across the $Z$ point for $\mathrm{K}_{0.77} \mathrm{Fe}_{1.65} \mathrm{Se}_{2}$, taken with $31-\mathrm{eV}$ photons in mixed polarization. (d) The photoemission intensity distribution along the $Z-A$ direction for $\mathrm{K}_{0.77} \mathrm{Fe}_{1.65} \mathrm{Se}_{2}$, taken with $121-\mathrm{eV}$ photons in $s$ polarization. Note that both $31-\mathrm{eV}$ and $121-\mathrm{eV}$ photons correspond to the $Z$ point in the Brillouin zone for $\mathrm{K}_{0.77} \mathrm{Fe}_{1.65} \mathrm{Se}_{2}$.
$\mathrm{K}_{0.77} \mathrm{Fe}_{1.65} \mathrm{Se}_{2}$. If we compare the electronic structure of NC32 with the band calculation of NaFeAs after a rigid band shift, we could get a renormalization factor of around 1.8 for NC32, which is smaller than both the factor of about 4 in $\mathrm{NaFeAs}$ and the factor of about 3 in $\mathrm{K}_{0.77} \mathrm{Fe}_{1.65} \mathrm{Se}_{2}$ (Refs. [62,63]). This implies that the superconductivity in $\mathrm{K}_{0.77} \mathrm{Fe}_{1.65} \mathrm{Se}_{2}$ and the monolayer FeSe thin film whose Fermi surfaces consist of only electron pockets could be supported by moderate electronic correlations.

The comparison between $\mathrm{NC} 32$ and $\mathrm{K}_{0.77} \mathrm{Fe}_{1.65} \mathrm{Se}_{2}$ proves that the same Fermi-surface topology could give dramatically different $T_{C}$ 's. Another similar example is that of $\mathrm{LC} 17$ and $\mathrm{Ca}_{10}\left(\mathrm{Pt}_{4} \mathrm{As}_{8}\right)\left(\mathrm{Fe}_{2-x} \mathrm{Pt}_{x} \mathrm{As}_{2}\right)_{5}$ - they have a similar Fermi-surface topology, with a $d_{x y}$-based hole pocket and an electron pocket around the zone center (Fig. 8) [64]; however, the $T_{C}$ is $22 \mathrm{~K}$ for $\mathrm{Ca}_{10}\left(\mathrm{Pt}_{4} \mathrm{As}_{8}\right)\left(\mathrm{Fe}_{2-x} \mathrm{Pt}_{x} \mathrm{As}_{2}\right)_{5}$ while it is $0 \mathrm{~K}$ for LC17. These comparisons indicate that superconductivity does not rely on the presence of the $d_{x z} / d_{y z}$-based hole pocket around the zone center, or even the presence of the hole pocket at all. On the other hand, completely different Fermi-surface topologies can host superconductivity of a similar strength. As shown in Fig. 8, the superconductivity could emerge on a Fermi surface consisting of only electron pockets $\left(\mathrm{K}_{0.77} \mathrm{Fe}_{1.65} \mathrm{Se}_{2}\right.$ and $\left.1 \mathrm{ML} \mathrm{FeSe} / \mathrm{STO}\right)$, only hole

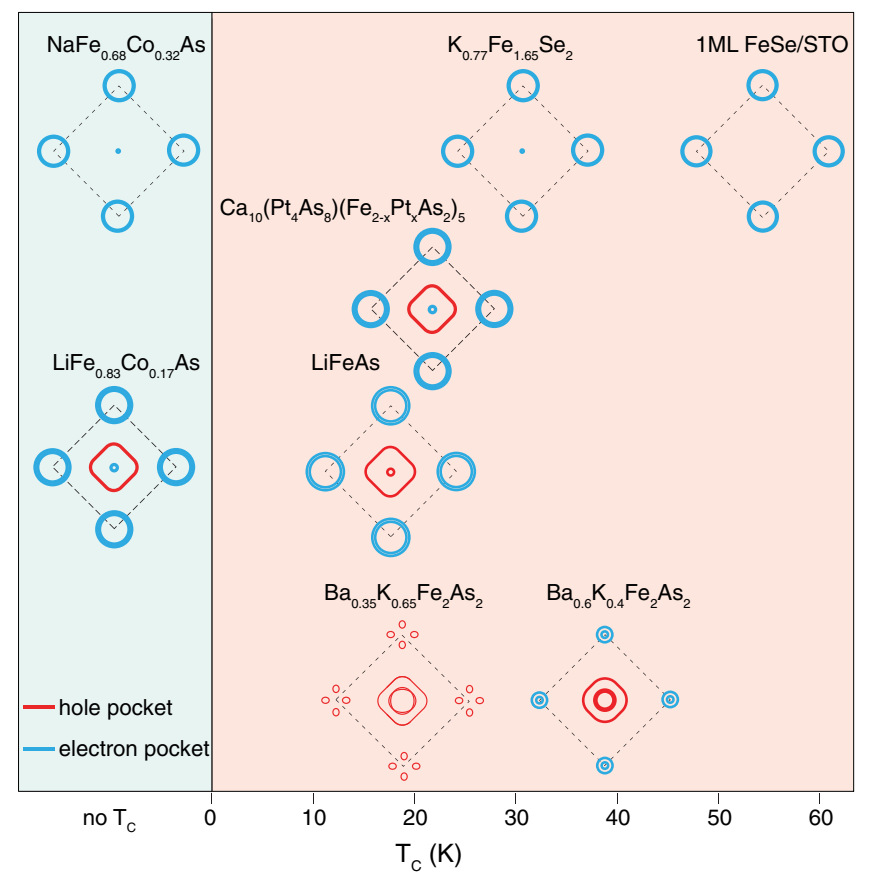

FIG. 8. Summary of the relation between Fermi-surface topology and $T_{C}$ for different compounds in FeHTS's. All the Fermi surfaces were taken across the $\Gamma$ point. The hole pockets and electron pockets are illustrated with red and blue lines, respectively. $T_{C}$ is not directly correlated with the Fermi-surface topology. The Fermi surfaces of $\mathrm{K}_{0.77} \mathrm{Fe}_{1.65} \mathrm{Se}_{2}$, $1 \mathrm{ML}$ $\mathrm{FeSe} / \mathrm{STO}$, and $\mathrm{Ca}_{10}\left(\mathrm{Pt}_{4} \mathrm{As}_{8}\right)\left(\mathrm{Fe}_{2-x} \mathrm{Pt}_{x} \mathrm{As}_{2}\right)_{5}$ were extracted from Refs. [19,20,64], respectively. 
pockets $\left(\mathrm{Ba}_{0.35} \mathrm{~K}_{0.65} \mathrm{Fe}_{2} \mathrm{As}_{2}\right)$, both hole and electron pockets ( $\mathrm{LiFeAs}$ and $\mathrm{Ba}_{0.6} \mathrm{~K}_{0.4} \mathrm{Fe}_{2} \mathrm{As}_{2}$ et al.) or with some special Fermi-surface forms [such as $\mathrm{Ca}_{10}\left(\mathrm{Pt}_{4} \mathrm{As}_{8}\right)\left(\mathrm{Fe}_{2-x} \mathrm{Pt}_{x} \mathrm{As}_{2}\right)_{5}$ with both hole and electron pockets around the zone center].

Considering all the facts given above, we conclude that the Fermi-surface topology may just play a secondary role in determining $T_{C}$. Other factors, such as the electronic correlation strength and impurity scattering discussed in the last two subsections, could play more important roles. We also note that, when the impurity scattering and the electronic correlation are both less sensitive to the dopants, as is the case in $\mathrm{Ba}_{1-x} \mathrm{~K}_{x} \mathrm{Fe}_{2} \mathrm{As}_{2}$ [Figs. 2(g) and 5(c)], the Fermi surface might play the leading role in determining $T_{C}$. As shown in Fig. 4, for $\mathrm{Ba}_{1-x} \mathrm{~K}_{x} \mathrm{Fe}_{2} \mathrm{As}_{2}$, the $T_{C}$ decreases much more slowly in the overdoped regime of the phase diagram compared with the other systems. The suppression of $T_{C}$ in $\mathrm{Ba}_{1-x} \mathrm{~K}_{x} \mathrm{Fe}_{2} \mathrm{As}_{2}$ was proposed to be due to the competition between the $s$-wave and $d$-wave pairing channels in the heavily doped compounds [65], whose strengths depend on the Fermi-surface topology.

\section{CONCLUSIONS}

To summarize, out of the diversified materials and electronic structures of various series of FeHTS's, we have uncovered a unifying theme of the doping effects: the bandwidth enhancement or electronic correlation suppression by both heterovalent and isovalent dopants, and the band-selective and site-dependent impurity scattering effects, for the first time. Together with the Fermi-surface alteration, these provide a microscopic and comprehensive understanding of chemical substitution in FeHTS's.

Particularly, we identified the pivotal role of the bandwidth or electronic correlation on the superconductivity in FeHTS's, which can serve as a more relevant and robust parameter than the different types of dopings, and thus provides a natural understanding of the similar phase diagrams obtained by various dopants. We further demonstrated that the electronic correlation in FeHTS's is closely related to both the carrier type of dopants and the lattice structure parameters, such as bond length. The different impurity scattering effects and different structures may affect the maximal value of $T_{C}$ and cause the superficial diversity and complexity. However, Fermi-surface topology and its evolution with doping may play a secondary role in determining $T_{C}$.

The implications of our experimental findings are manyfold. They explain many puzzles and controversies and provide a new view for understanding the phase diagrams, resistivity behaviors, superconducting properties, etc. Our data also suggest that one needs to minimize the impurity scattering in the Fe-anion layer while optimizing a moderate electronic correlation in order to enhance the $T_{C}$ record in the search for new FeHTS's. Furthermore, these results put strong constraints on the theories of the superconducting mechanism in FeHTS's. As the $T_{C}$ is less sensitive to the Fermi-surface topology, the weakcoupling theories based on the Fermi surface should be reexamined [50,51]. Alternatively, the strong-coupling pairing scenario, where the superconducting pairing is mediated by the local antiferromagnetic exchange interactions or spin fluctuations, is favored since they are sensitive to the electronic correlations instead of the Fermi-surface topology [66].

\section{ACKNOWLEDGMENTS}

We gratefully acknowledge experimental support from Dr. D. H. Lu, Dr. H. Makoto at SSRL, Dr. M. Shi, Dr. N. Plumb at SLS, Professor K. Shimada, Dr. M. Arita, and Dr. J. Jiang at HiSOR, as well as helpful discussions with Professor Jiangping $\mathrm{Hu}$ and Professor Zhongyi Lu. This work is supported in part by the National Science Foundation of China and the National Basic Research Program of China (973 Program) under Grants No. 2012CB921402, No. 2011CBA00112, and No. 2011CB921802. SSRL is operated by the US DOE, Office of BES, Divisions of Chemical Sciences and Material Sciences.

[1] J. Kondo, Resistance Minimum in Dilute Magnetic Alloys, Prog. Theor. Phys. 32, 37 (1964).

[2] M. Imada, A. Fujimori, and Y. Tokura, Metal-Insulator Transitions, Rev. Mod. Phys. 70, 1039 (1998).

[3] H. C. Xu, Y. Zhang, M. Xu, R. Peng, X. P. Shen, V. N. Strocov, M. Shi, M. Kobayashi, T. Schmitt, B. P. Xie, and D. L. Feng, Direct Observation of the Bandwidth Control Mott Transition in the $\mathrm{NiS}_{2-x} \mathrm{Se}_{x}$ Multiband System, Phys. Rev. Lett. 112, 087603 (2014).

[4] J. Paglione and R. L. Greene, High-Temperature Superconductivity in Iron-Based Materials, Nat. Phys. 6, 645 (2010).

[5] G. R. Stewart, Superconductivity in Iron Compounds, Rev. Mod. Phys. 83, 1589 (2011).

[6] H. Chen, Y. Ren, Y. Qiu, Wei Bao, R. H. Liu, G. Wu, T. Wu, Y.L. Xie, X.F. Wang, Q. Huang, and X. H. Chen, Coexistence of the Spin-Density Wave and Superconductivity in $\mathrm{Ba}_{1-x} \mathrm{~K}_{x} \mathrm{Fe}_{2} \mathrm{As}_{2}$, Europhys. Lett. 85, 17006 (2009).

[7] D. K. Pratt, W. Tian, A. Kreyssig, J. L. Zarestky, S. Nandi, N. Ni, S. L. Bud'ko, P. C. Canfield, A. I. Goldman, and R. J. McQueeney, Coexistence of Competing Antiferromagnetic and Superconducting Phases in the Underdoped $\mathrm{Ba}\left(\mathrm{Fe}_{0.953} \mathrm{Co}_{0.047}\right){ }_{2} \mathrm{As}_{2}$ Compound Using X-ray and Neutron Scattering Techniques, Phys. Rev. Lett. 103, 087001 (2009).

[8] S. Kasahara, T. Shibauchi, K. Hashimoto, K. Ikada, S. Tonegawa, R. Okazaki, H. Shishido, H. Ikeda, H. Takeya, K. Hirata, T. Terashima, and Y. Matsuda, Evolution from Non-Fermi- to Fermi-Liquid Transport via Isovalent Doping in $\mathrm{BaFe}_{2}\left(\mathrm{As}_{1-x} \mathrm{P}_{x}\right)_{2}$ Superconductors, Phys. Rev. B 81, 184519 (2010). 
[9] Z. R. Ye, Y. Zhang, F. Chen, M. Xu, Q. Q. Ge, J. Jiang, B. P. Xie, and D. L. Feng, Doping Dependence of the Electronic Structure in Phosphorus-Doped Ferropnictide Superconductor $\mathrm{BaFe}_{2}\left(\mathrm{As}_{1-x} \mathrm{P}_{x}\right)_{2}$ Studied by AngleResolved Photoemission Spectroscopy, Phys. Rev. B 86, 035136 (2012).

[10] M. J. Eom, S. W. Na, C. Hoch, R. K. Kremer, and J. S. Kim, Evolution of Transport Properties of $\mathrm{BaFe}_{2-x} \mathrm{Ru}_{x} \mathrm{As}_{2}$ in a Wide Range of Isovalent Ru Substitution, Phys. Rev. B 85, 024536 (2012).

[11] A. Damascelli, Z. Hussain, and Z.-X. Shen, Angle-Resolved Photoemission Studies of the Cuprate Superconductors, Rev. Mod. Phys. 75, 473 (2003).

[12] T. J. Liu, J. Hu, B. Qian, D. Fobes, Z. Q. Mao, W. Bao, M. Reehuis, S. A. J. Kimber, K. Proke, S. Matas, D. N. Argyriou, A. Hiess, A. Rotaru, H. Pham, L. Spinu, Y. Qiu, V. Thampy, A. T. Savici, J. A. Rodriguez, and C. Broholm, From $(\pi, 0)$ Magnetic Order to Superconductivity with $(\pi, \pi)$ Magnetic Resonance in $\mathrm{Fe}_{1.02} \mathrm{Te}_{1-x} \mathrm{Se}_{x}$, Nat. Mater. 9, 718 (2010).

[13] D. R. Parker, M. J. P. Smith, T. Lancaster, A. J. Steele, I. Franke, P. J. Baker, F. L. Pratt, M. J. Pitcher, S. J. Blundell, and S.J. Clarke, Control of the Competition between a Magnetic Phase and a Superconducting Phase in Cobalt-Doped and Nickel-Doped NaFeAs Using Electron Count, Phys. Rev. Lett. 104, 057007 (2010).

[14] M. J. Pitcher, T. Lancaster, J. D. Wright, I. Franke, A. J. Steele, P. J. Baker, F. L. Pratt, W. T. Thomas, D. R. Parker, S. J. Blundell, and S. J. Clarke, Compositional Control of the Superconducting Properties of LiFeAs, J. Am. Chem. Soc. 132, 10467 (2010).

[15] Y. Kamihara, T. Watanabe, M. Hirano, and H. Hosono, Iron-Based Layered Superconductor $\mathrm{La}\left[\mathrm{O}_{1-x} \mathrm{~F}_{x}\right] \mathrm{FeAs}$ $(x=0.05-0.12)$ with $T_{C}=26 \mathrm{~K}$, J. Am. Chem. Soc. 130, 3296 (2008).

[16] S. Iimura, S. Matsuishi, H. Sato, T. Hanna, Y. Muraba, S. W. Kim, J. E. Kim, M. Takata, and H. Hosono, Two-Dome Structure in Electron-Doped Iron Arsenide Superconductors, Nat. Commun. 3, 943 (2012).

[17] C. Liu, A. D. Palczewski, R. S. Dhaka, T. Kondo, R. M. Fernandes, E. D. Mun, H. Hodovanets, A. N. Thaler, J. Schmalian, S. L. Bud'ko, P.C. Canfield, and A. Kaminski, Importance of the Fermi-Surface Topology to the Superconducting State of the Electron-Doped Pnictide $\mathrm{Ba}\left(\mathrm{Fe}_{1-x} \mathrm{Co}_{x}\right)_{2} \mathrm{As}_{2}$, Phys. Rev. B 84, 020509 (2011).

[18] P. Richard, T. Sato, K. Nakayama, T. Takahashi, and H. Ding, Fe-Based Superconductors: An Angle-Resolved Photoemission Spectroscopy Perspective, Rep. Prog. Phys. 74, 124512 (2011).

[19] Y. Zhang, L. X. Yang, M. Xu, Z. R. Ye, F. Chen, C. He, H. C. Xu, J. Jiang, B. P. Xie, J. J. Ying, X. F. Wang, X. H. Chen, J. P. Hu, M. Matsunami, S. Kimura, and D. L. Feng, Nodeless Superconducting Gap in $\mathrm{A}_{x} \mathrm{Fe}_{2} \mathrm{Se}_{2}(\mathrm{~A}=\mathrm{K}, \mathrm{Cs})$ Revealed by Angle-Resolved Photoemission Spectroscopy, Nat. Mater. 10, 273 (2011).

[20] S. Y. Tan, M. Xia, Y. Zhang, Z. R. Ye, F. Chen, X. Xie, R. Peng, D. F. Xu, Q. Fan, H. C. Xu, J. Juan, T. Zhang, X. C. Lai, T. Xiang, J. P. Hu, B. P. Xie, and D. L. Feng, InterfaceInduced Superconductivity and Strain-Dependent Spin
Density Waves in $\mathrm{FeSe} / \mathrm{SrTiO}_{3}$ Thin Films, Nat. Mater. 12, 634 (2013).

[21] S. He, J. He, W. Zhang, L. Zhao, D. Liu, X. Liu, D. Mou, Y.-B. Ou, Q.-Y. Wang, Z. Li, L. Wang, Y. Peng, Y. Liu, C. Chen, L. Yu, G. Liu, X. Dong, J. Zhang, C. Chen, Z. Xu, X. Chen, X. Ma, Q. Xue, and X. J. Zhou, Phase Diagram and Electronic Indication of High-Temperature Superconductivity at $65 \mathrm{~K}$ in Single-Layer FeSe Films, Nat. Mater. 12, 605 (2013).

[22] K. Fujita, T. Noda, K. M. Kojima, H. Eisaki, and S. Uchida, Effect of Disorder Outside the $\mathrm{CuO}_{2}$ Planes on $T_{C}$ of Copper Oxide Superconductors, Phys. Rev. Lett. 95, 097006 (2005).

[23] K. Kirshenbaum, S. R. Saha, S. Ziemak, T. Drye, and J. Paglione, Universal Pair-Breaking in TransitionMetal-Substituted Iron-Pnictide Superconductors, Phys. Rev. B 86, 140505 (2012).

[24] A. S. Sefat, R. Jin, M. A. McGuire, B. C. Sales, D. J. Singh, and D. Mandrus, Superconductivity at $22 \mathrm{~K}$ in Co-Doped $\mathrm{BaFe}_{2} \mathrm{As}_{2}$ Crystals, Phys. Rev. Lett. 101, 117004 (2008).

[25] Y. Wang, A. Kreisel, P. J. Hirschfeld, and V. Mishra, Using Controlled Disorder to Distinguish $s \pm$ and $s++$ Gap Structure in Fe-Based Superconductors, Phys. Rev. B 87, 094504 (2013).

[26] P. J. Hirschfeld, M. M. Korshunov, and I. I. Mazin, Gap Symmetry and Structure of Fe-Based Superconductors, Rep. Prog. Phys. 74, 124508 (2011).

[27] C.-H. Lee, A. Iyo, H. Eisaki, H. Kito, M. T. Fernandez-Diaz, T. Ito, K. Kihou, H. Matsuhata, M. Braden, and K. Yamada, Effect of Structural Parameters on Superconductivity in Fluorine-Free $\mathrm{LnFeAsO}_{1-y}(\mathrm{Ln}=\mathrm{La}, \mathrm{Nd})$, J. Phys. Soc. Jpn. 77, 083704 (2008).

[28] Y. Mizuguhci, Y. Hara, K. Deguchi, S. Tsuda, T. Yamaguchi, K. Takeda, H. Kotegawa, H. Tou, and Y. Takano, Anion Height Dependence of $T_{C}$ for the Fe-Based Superconductor, Supercond. Sci. Technol. 23, 054013 (2010).

[29] S. V. Borisenko, V. B. Zabolotnyy, D. V. Evtushinsky, T. K. Kim, I. V. Morozov, A. N. Yaresko, A. A. Kordyuk, G. Behr, A. Vasiliev, R. Follath, and B. Büchner, Superconductivity without Nesting in LiFeAs, Phys. Rev. Lett. 105, 067002 (2010).

[30] M. Nakajima, T. Tanaka, S. Ishida, K. Kihou, C. H. Lee, A. Iyo, T. Kakeshita, H. Eisaki, and S. Uchida, Crossover from Bad to Good Metal in $\mathrm{BaFe}_{2}\left(\mathrm{As}_{1-x} \mathrm{P}_{x}\right)_{2}$ Induced by Isovalent P Substitution, Phys. Rev. B 88, 094501 (2013).

[31] F. Chen, B. Zhou, Y. Zhang, J. Wei, H.-W. Ou, J.-F. Zhao, C. He, Q.-Q. Ge, M. Arita, K. Shimada, H. Namatame, M. Taniguchi, Z.-Y. Lu, J. Hu, X.-Y. Cui, and D. L. Feng, Electronic Structure of $\mathrm{Fe}_{1.04} \mathrm{Te}_{0.66} \mathrm{Se}_{0.34}$, Phys. Rev. B 81, 014526 (2010).

[32] J. J. Ying, X. F. Wang, X. G. Luo, A. F. Wang, M. Zhang, Y. J. Yan, Z. J. Xiang, R. H. Liu, P. Cheng, G. J. Ye, and X. H. Chen, Superconductivity and Magnetic Properties of Single Crystals of $\mathrm{K}_{0.75} \mathrm{Fe}_{1.66} \mathrm{Se}_{2}$ and $\mathrm{Cs}_{0.81} \mathrm{Fe}_{1.61} \mathrm{Se}_{2}$, Phys. Rev. B 83, 212502 (2011).

[33] T. Hajiri, T. Ito, R. Niwa, M. Matsunami, B. H. Min, Y. S. Kwon, and S. Kimura, Three-Dimensional Electronic Structure and Interband Nesting in the Stoichiometric Superconductor LiFeAs, Phys. Rev. B 85, 094509 (2012). 
[34] V. Vildosola, L. Pourovskii, R. Arita, S. Biermann, and A. Georges, Bandwidth and Fermi Surface of Iron Oxypnictides: Covalency and Sensitivity to Structural Changes, Phys. Rev. B 78, 064518 (2008).

[35] A. Subedi, L. Zhang, D. J. Singh, and M. H. Du, Density Functional Study of FeS, FeSe, and FeTe: Electronic Structure, Magnetism, Phonons, and Superconductivity, Phys. Rev. B 78, 134514 (2008).

[36] K. Suzuki, H. Usui, and K. Kuroki, Possible ThreeDimensional Nodes in the $s$ Superconducting Gap of $\mathrm{BaFe}_{2}\left(\mathrm{As}_{1-x} \mathrm{P}_{x}\right)_{2}$, J. Phys. Soc. Jpn. 80, 013710 (2011).

[37] W. Li, J. Li, J.-X. Zhu, Y. Chen, and C. S. Ting, Possible Pairing Symmetry in Iron-Pnictide Superconductor $\mathrm{KFe}_{2} \mathrm{As}_{2}$, Europhys. Lett. 99, 57006 (2012).

[38] J. Maletz, V. B. Zabolotnyy, D. V. Evtushinsky, S. Thirupathaiah, A. U. B. Wolter, L. Harnagea, A. N. Yaresko, A. N. Vasiliev, D. A. Chareev, E. D. L. Rienks, B. Büchner, and S. V. Borisenko, Unusual Band Renormalization in the Simplest Iron Based Superconductor, Phys. Rev. B 89, 220506(R) (2014).

[39] K. Nakayama, Y. Miyata, G. N. Phan, T. Sato, Y. Tanabe, T. Urata, K. Tanigaki, and T. Takahashi, Reconstruction of Band Structure Induced by Electronic Nematicity in FeSe Superconductor, arXiv:1404.0857 [Phys. Rev. Lett. (to be published)].

[40] Y. Mizuguchi and Y. Takano, Review of Fe Chalcogenides as the Simplest Fe-Based Superconductor, J. Phys. Soc. Jpn. 79, 102001 (2010).

[41] A. A. Kordyuk, V. B. Zabolotnyy, D. V. Evtushinsky, A. N. Yaresko, B. Büchner, and S. V. Borisenko, Electronic Band Structure of Ferro-Pnictide Superconductors from ARPES Experiment, J. Supercond. Novel Magn. 26, 2837 (2013).

[42] H. Usui and K. Kuroki, Maximizing the Fermi-Surface Multiplicity Optimizes the Superconducting State of Iron Pnictide Compounds, Phys. Rev. B 84, 024505 (2011).

[43] Z.-A. Ren, W. Lu, J. Yang, W. Yi, X.-L. Shen, C. Zheng, G.-C. Che, X.-L. Dong, L.-L. Sun, F. Zhou, and Z.-X. Zhao, Superconductivity at $55 \mathrm{~K}$ in Iron-Based F-Doped Layered Quaternary Compound $\operatorname{Sm}\left[\mathrm{O}_{1-x} \mathrm{~F}_{x}\right]$ FeAs, Chin. Phys. Lett. 25, 2215 (2008).

[44] H. Yang, Z. Wang, D. Fang, S. Li, T. Kariyado, G. Chen, M. Ogata, T. Das, A. V. Balatsky, and H.-H. Wen, Unexpected Weak Spatial Variation in the Local Density of States Induced by Individual Co Impurity Atoms in Superconducting $\mathrm{Na}\left(\mathrm{Fe}_{1-x} \mathrm{Co}_{x}\right)$ As Crystals Revealed by Scanning Tunneling Spectroscopy, Phys. Rev. B 86, 214512 (2012).

[45] S. Sharma, A. Bharathi, K. Vinod, C. S. Sundar, V. Srihari, S. Sen, H. Ghosh, A. K. Sinha, and S. K. Deb, Structural Investigations in $\mathrm{BaFe}_{2-x} \mathrm{Ru}_{x} \mathrm{As}_{2}$ as a Function of $\mathrm{Ru}$ and Temperature, arXiv:1312.7055.

[46] D. C. Johnston, The Puzzle of High Temperature Superconductivity in Layered Iron Pnictides and Chalcogenides, Adv. Phys. 59, 803 (2010).

[47] Y. Zhang, F. Chen, C. He, L. X. Yang, B. P. Xie, Y. L. Xie, X. H. Chen, M. Fang, M. Arita, K. Shimada, H. Namatame, M. Taniguchi, J. P. Hu, and D. L. Feng, Strong Correlations and Spin-Density-Wave Phase Induced by a Massive Spectral Weight Redistribution in $\alpha-\mathrm{Fe}_{1.06}$ Te, Phys. Rev. B 82, 165113 (2010).
[48] Z. P. Yin, K. Haule, and G. Kotliar, Kinetic Frustration and the Nature of the Magnetic and Paramagnetic States in Iron Pnictides and Ironchalcogenides, Nat. Mater. 10, 932 (2011).

[49] T. Misawa, K. Nakamura, and M. Imada, Ab Initio Evidence for Strong Correlation Associated with Mott Proximity in Iron-Based Superconductors, Phys. Rev. Lett. 108, 177007 (2012).

[50] K. Kuroki, S. Onari, R. Arita, H. Usui, Y. Tanaka, H. Kontani, and H. Aoki, Unconventional Pairing Originating from the Disconnected Fermi Surfaces of Superconducting $\mathrm{LaFeAsO}_{1-x} \mathrm{~F}_{x}$, Phys. Rev. Lett. 101, 087004 (2008).

[51] I. I. Mazin, D. J. Singh, M. D. Johannes, and M. H. Du, Unconventional Superconductivity with a Sign Reversal in the Order Parameter of $\mathrm{LaFeAsO}_{1-x} \mathrm{~F}_{x}$, Phys. Rev. Lett. 101, 057003 (2008).

[52] T. Saito, S. Onari, and H. Kontani, Orbital Fluctuation Theory in Iron Pnictides: Effects of As-Fe-As Bond Angle, Isotope Substitution, and $Z^{2}$-Orbital Pocket on Superconductivity, Phys. Rev. B 82, 144510 (2010).

[53] H. Shishido, A. F. Bangura, A. I. Coldea, S. Tonegawa, K. Hashimoto, S. Kasahara, P. M. C. Rourke, H. Ikeda, T. Terashima, R. Settai, Y. Onuki, D. Vignolles, C. Proust, B. Vignolle, A. McCollam, Y. Matsuda, T. Shibauchi, and A. Carrington, Evolution of the Fermi Surface of $\mathrm{BaFe}_{2}\left(\mathrm{As}_{1-x} \mathrm{P}_{x}\right)_{2}$ on Entering the Superconducting Dome, Phys. Rev. Lett. 104, 057008 (2010).

[54] D. H. Lu, M. Yi, S. K. Mo, A. S. Erickson, J. Analytis, J. H. Chu, D. J. Singh, Z. Hussain, T. H. Geballe, I. R. Fisher, and Z.X. Shen, Electronic Structure of the Iron-Based Superconductor LaOFeP, Nature (London) 455, 81 (2008).

[55] G. F. Chen, Z. G. Chen, J. Dong, W. Z. Hu, G. Li, X. D. Zhang, P. Zheng, J. L. Luo, and N. L. Wang, Electronic Properties of Single-Crystalline $\mathrm{Fe}_{1.05} \mathrm{Te}$ and $\mathrm{Fe}_{1.03} \mathrm{Te}_{0.3} \mathrm{Se}_{0.7}$, Phys. Rev. B 79, 140509(R) (2009).

[56] S. Li, C. de la Cruz, Q. Huang, Y. Chen, J. W. Lynn, J. Hu, Y.-L. Huang, F.-C. Hsu, K.-W. Yeh, M.-K. Wu, and P. Dai, First-Order Magnetic and Structural Phase Transitions in $\mathrm{Fe}_{1+y} \mathrm{Se}_{x} \mathrm{Te}_{1-x}$, Phys. Rev. B 79, 054503 (2009).

[57] S. T. Cui, S. Y. Zhu, A. F. Wang, S. Kong, S. L. Ju, X. G. Luo, X. H. Chen, G. B. Zhang, and Z. Sun, Evolution of the Band Structure of Superconducting NaFeAs from Optimally Doped to Heavily Overdoped Co Substitution Using Angle-Resolved Photoemission Spectroscopy, Phys. Rev. B 86, 155143 (2012).

[58] F. Chen, M. Xu, Q. Ge, Y. Zhang, Z. Ye, L. Yang, J. Jiang, B. Xie, R. Che, M. Zhang, A. Wang, X. Chen, D. Shen, J. $\mathrm{Hu}$, and D. Feng, Electronic Identification of the Parental Phases and Mesoscopic Phase Separation of $\mathrm{K}_{x} \mathrm{Fe}_{2-y} \mathrm{Se}_{2}$ Superconductors, Phys. Rev. X 1, 021020 (2011).

[59] W. Li, H. Ding, Z. Li, P. Deng, K. Chang, K. He, S. Ji, L. Wang, X. Ma, J.-P. Hu, X. Chen, and Q.-K. Xue, $\mathrm{KFe}_{2} \mathrm{Se}_{2}$ is the Parent Compound of K-Doped Iron Selenide Superconductors, Phys. Rev. Lett. 109, 057003 (2012).

[60] R. Peng, H. C. Xu, S. Y. Tan, M. Xia, X. P. Shen, Z. C. Huang, C. H. P. Wen, Q. Song, T. Zhang, B. P. Xie, and D. L. Feng, Critical Role of Substrate in the High Temperature Superconductivity of Single Layer FeSe on $\mathrm{Nb}: \mathrm{BaTiO}_{3}$, arXiv:1402.1357. 
[61] Y.-Y. Xiang, F. Wang, D. Wang, Q.-H. Wang, and D.-H. Lee, High-Temperature Superconductivity at the $\mathrm{FeSe} / \mathrm{SrTiO}_{3}$ Interface, Phys. Rev. B 86, 134508 (2012).

[62] S. Deng, J. Khler, and A. Simon, Electronic Structure and Lattice Dynamics of NaFeAs, Phys. Rev. B 80, 214508 (2009).

[63] X.-W. Yan, M. Gao, Z.-Y. Lu, and T. Xiang, Electronic and Magnetic Structures of the Ternary Iron Selenides $\mathrm{AFe}_{2} \mathrm{Se}_{2}$ (A = Cs, Rb, K, or Tl), Phys. Rev. B 84, 054502 (2011).

[64] X. P. Shen, S. D. Chen, Q. Q. Ge, Z. R. Ye, F. Chen, H. C. Xu, S. Y. Tan, X. H. Niu, Q. Fan, B. P. Xie, and D. L. Feng,
Electronic Structure of $\mathrm{Ca}_{10}\left(\mathrm{Pt}_{4} \mathrm{As}_{8}\right)\left(\mathrm{Fe}_{2-x} \mathrm{Pt}_{x} \mathrm{As}_{2}\right)_{5}$ with Metallic $\mathrm{Pt}_{4} \mathrm{As}_{8}$ Layers: An Angle-Resolved Photoemission Spectroscopy Study, Phys. Rev. B 88, 115124 (2013).

[65] F. F. Tafti, A. Juneau-Fecteau, M.-E. Delage, S. Rene de Cotret, J.-Ph. Reid, A. F. Wang, X.-G. Luo, X. H. Chen, N. Doiron-Leyraud, and Louis Taillefer, Sudden Reversal in the Pressure Dependence of $T_{C}$ in the Iron-Based Superconductor $\mathrm{KFe}_{2} \mathrm{As}_{2}$, Nat. Phys. 9, 349 (2013).

[66] J. Hu and H. Ding, Local Antiferromagnetic Exchange and Collaborative Fermi Surface as Key Ingredients of High Temperature Superconductors, Sci. Rep. 2, 381 (2012). 